\title{
TROPICAL TOTALLY POSITIVE MATRICES
}

\author{
STÉPHANE GAUBERT AND ADI NIV
}

\begin{abstract}
We investigate the tropical analogues of totally positive and totally nonnegative matrices. These arise when considering the images by the nonarchimedean valuation of the corresponding classes of matrices over a real nonarchimedean valued field, like the field of real Puiseux series. We show that the nonarchimedean valuation sends the totally positive matrices precisely to the Monge matrices. This leads to explicit polyhedral representations of the tropical analogues of totally positive and totally nonnegative matrices. We also show that tropical totally nonnegative matrices with a finite permanent can be factorized in terms of elementary matrices. We finally determine the eigenvalues of tropical totally nonnegative matrices, and relate them with the eigenvalues of totally nonnegative matrices over nonarchimedean fields.

Keywords: Total positivity; total nonnegativity; tropical geometry; compound matrix; permanent; Monge matrices; Grassmannian; Plücker coordinates.

AMSC: $15 A 15$ (Primary), 15A09, 15A18, 15A24, 15A29, 15A75, 15A80, $15 B 99$.
\end{abstract}

\section{INTRODUCTION}

1.1. Motivation and background. A real matrix is said to be totally positive (resp. totally nonnegative) if all its minors are positive (resp. nonnegative). These matrices arise in several classical fields, such as oscillatory matrices (see e.g. [And87, §4]), or approximation theory (see e.g. [GM96]); they have appeared more recently in the theory of canonical bases for quantum groups BFZ96. We refer the reader to the monograph of Fallat and Johnson in FJ11 or to the survey of Fomin and Zelevinsky [FZ00 for more information. Totally positive/nonnegative matrices can be defined over any real closed field, and in particular, over nonarchimedean fields, like the field of Puiseux series with real coefficients. In this paper, we characterize the set of images of such matrices by the nonarchimedean valuation which associates to a Puiseux series its leading exponent. To do so, we study the tropical analogues of totally positive and totally nonnegative matrices.

To describe further our results, it is convenient to recall some basic notions. The max-plus (or tropical) semifield, denoted by $\mathbb{R}_{\max }$, is the set $\mathbb{R} \cup\{-\infty\}$ equipped with the laws $a \oplus b:=\max (a, b)$ and $a \odot b:=a+b$. (See for instance BCOQ92, IMS07, ABG07, But10, MS15.) It has a zero element, $\mathbb{O}=-\infty$, and a unit element, $\mathbb{1}=0$. We abuse notation by using the same symbol, $\mathbb{R}_{\max }$ for the semifield and for its ground set. The tropical numbers can be thought of as the images by the valuation of the elements of a nonarchimedean field. A convenient choice of field, denoted by $\mathbb{K}$, consists of (generalized) Puiseux series with real coefficients and real exponents. Such a series can be written as

$$
f:=\sum_{k \geqslant 0} a_{k} t^{b_{k}}
$$

where $a_{k} \in \mathbb{R}, b_{k} \in \mathbb{R}$, and $\left(b_{k}\right)$ is a decreasing sequence converging to $-\infty$. The valuation of $\boldsymbol{f}$ is defined to be the largest exponent of $\boldsymbol{f}$, i.e., $\operatorname{val}(\boldsymbol{f}):=\sup \left\{b_{k} \mid a_{k} \neq 0\right\}$, with the convention that $\operatorname{val}(0)=-\infty$.

The first author has been partially supported by the Gaspard Monge Program (PGMO) of FMJH and EDF, by a public grant as part of the Investissement d'avenir project, reference ANR-11-LABX-0056-LMH, LabEx LMH, and by the MALTHY Project of the ANR Program. The second author was sported by the French Chateaubriand grant and INRIA postdoctoral fellowship.

We thank Gleb Koshevoy for suggesting to investigate the present topic and for discussions. We also thank Charles Johnson for pointing out the interpretation of our results in terms of Monge matrices, and Benjamin Schröter for helpful comments. 
The choice of this specific field of formal series is only to keep the exposition concrete. What matters is that the nonarchimedean field is real closed, that its value group is $\mathbb{R}$, and that its valuation has a certain property called convexity; we refer the reader to Section 2 for more details on the setting in which our results hold. We emphasize in particular that our main results also apply to fields of absolutely convergent series with real exponents like the ones of vdDS98 and more generally to Hardy fields of polynomially bounded o-minimal structures [Ale13.

A nonzero series is said to be positive if its leading coefficient is positive. We denote by $\mathbb{K}_{>0}$ the set of positive series, and we denote by $\mathbb{K}_{\geqslant 0}:=\mathbb{K}_{>0} \cup\{0\}$ the set of nonnegative series. The map val satisfies, for all $\boldsymbol{f}, \boldsymbol{g} \in \mathbb{K}_{\geqslant 0}$,

$$
\operatorname{val}(\boldsymbol{f}+\boldsymbol{g})=\max (\operatorname{val}(\boldsymbol{f}), \operatorname{val}(\boldsymbol{g})), \quad \operatorname{val}(\boldsymbol{f g})=\operatorname{val}(\boldsymbol{f})+\operatorname{val}(\boldsymbol{g}) .
$$

In tropical algebra, we are interested in properties of objects defined over $\mathbb{K}$ that can be inferred from this valuation. We study here the images by the valuation of the classical classes of totally positive or totally nonnegative matrices over $\mathbb{K}$. To do so, we associate to a $d \times d$ matrix $\boldsymbol{A}=\left(\boldsymbol{A}_{i, j}\right)$ with entries in $\mathbb{K}_{\geqslant 0}$, the matrix $A$ with entries $A_{i j}:=\operatorname{val}\left(\boldsymbol{A}_{i, j}\right)$ in $\mathbb{R}_{\text {max }}$. We say that $\boldsymbol{A}_{i, j}$ is a lift of $A_{i, j}$, and $\boldsymbol{A}$ is a lift of $A$, with the convention that 0 is the lift of $-\infty$. The tropical permanent of $A$ is defined as

$$
\operatorname{per}(A):=\max _{\sigma \in S_{d}} \sum_{i \in[d]} A_{i, \sigma(i)}
$$

where $S_{d}$ is the set of permutations on $[d]:=\{1, \ldots, d\}$, and $\sum_{i \in[d]} A_{i, \sigma(i)}$ is the weight of the permutation $\sigma$ in $\operatorname{per}(A)$.

We say that the matrix $A$ is (tropically) sign-nonsingular if $\operatorname{per}(A) \neq-\infty$ and if all the permutations $\sigma$ of maximum weight have the same parity. Otherwise, $A$ is said to be (tropically) sign-singular. We refer the reader to [BS95] for more background on the classical notion of sign-nonsingularity, and to [GB99, ABGJ15] for its tropical version. When $A$ is sign-nonsingular, it is easily seen that

$$
\operatorname{val}(\operatorname{det}(\boldsymbol{A}))=\operatorname{per}(A),
$$

and the sign of $\operatorname{det}(\boldsymbol{A})$ coincide with the sign of every permutation of maximal weight in $\operatorname{per}(A)$. A tropical minor is defined as the tropical permanent of a square submatrix. A tropical minor is said to be tropically positive (resp. tropically negative) if all its permutations of maximum weight are even (resp. odd). It is said to be tropically nonnegative (resp. tropically nonpositive) if either the above condition holds or the submatrix is sign-singular. This terminology can be justified by embedding the max-plus semiring in the symmetrized max-plus semiring [Plu90, AGG14].

A tropical matrix is said to be tropical totally positive if its entries are in $\mathbb{R}$, and all its minors are tropically positive. It is said to be tropical totally nonnegative if its entries are in $\mathbb{R}_{\max }$, and all its minors are tropically nonnegative.

Summary of notation. It is convenient now to list the main notations used in the manuscript. We follow the notation used by Fallat and Johnson [FJ11] for various classes of totally nonnegative matrices.

We denote by TP (resp. TN) the set of totally positive (resp. totally nonnegative) matrices over a field. We write $\operatorname{TP}(\mathbb{K})$ or $\operatorname{TP}(\mathbb{R})$, indicating the ground field in parenthesis, when necessary, and we use a similar notation for $\mathrm{TN}$. The set $\mathrm{TP}_{t}\left(\right.$ resp. $\left.\mathrm{TN}_{t}\right)$ denotes matrices whose minors of size at most $t$ are positive (resp. nonnegative). Similarly, we shall denote by TP ${ }^{\text {trop }}$ (resp. TN ${ }^{\text {trop }}$ ) the set of tropical totally positive (resp. tropical totally nonnegative) matrices, which have entries in $\mathbb{R}_{\max }$. In general the entries of a matrix in $T N^{\text {trop }}$ may take the $-\infty$ value. We denote by $\operatorname{TN}^{\text {trop }}(\mathbb{R})$ the subset of matrices in $\mathrm{TN}^{\text {trop }}$ whose entries belong to $\mathbb{R}$. We also denote by $\mathrm{TP}_{t}^{\text {trop }}$ (resp. $\mathrm{TN}_{t}^{\text {trop }}$ ) the set of matrices with entries in $\mathbb{R}_{\max }$ whose every tropical minor of size at most $t$ is tropically positive (resp. tropically nonnegative). Moreover, $\mathrm{TN}_{t}^{\text {trop }}(\mathbb{R})$ denotes the subset of $\mathrm{TN}_{t}^{\text {trop }}$ consisting of matrices with entries in $\mathbb{R}$.

We also denote by $D^{\text {trop }}$ the set of matrices such that every submatrix is tropical diagonally dominant. In this setting, a matrix $A$ is said to be tropical diagonally dominant if $\operatorname{per}(A)=\sum_{i \in[n]} A_{i, i}$. If $\operatorname{per}(A)>\sum_{i \in[n]} A_{i, \sigma(i)}$ for all permutations $\sigma$ distinct from the identity, the matrix $A$ is said to be tropical strictly diagonally dominant; the set of such matrices is denoted by SDD ${ }^{\text {trop }}$. 
We draw the reader's attention to the notation TP, used in FJ11] to denote totally positive matrices. This should not be confused with the notation $\mathbb{T P}^{n-1}$, used in [DS04] to denote the stratum of the $(n-1)$-dimensional tropical projective space that consists of rays generated by finite vectors; here we use the notation $\mathbb{P}^{n-1}\left(\mathbb{R}_{\max }\right)$ for the tropical projective space.

1.2. Main results. Our main results relate the classical and tropical notions of total nonnegativity. The following theorem follows by combining Theorem 3.4. Corollary 5.9 and Corollary 5.13 below, it shows that the image by the valuation of the set of tropical total positive matrices is determined by the tropical positivity of $2 \times 2$ minors.

Theorem A. We have

$$
\mathrm{TN}_{2}^{\text {trop }}(\mathbb{R})=\mathrm{TN}^{\text {trop }}(\mathbb{R})=\operatorname{val}\left(\mathrm{TN}\left(\mathbb{K}^{*}\right)\right)=\operatorname{val}(\mathrm{TP}) .
$$

We shall see that $\mathrm{TN}_{2}^{\text {trop }}(\mathbb{R})$ is precisely the set of Monge matrices, named after Gaspard Monge, as they arise in optimal transportation problems. The set of Monge matrices has an explicit polyhedral parametrization which follows from results of [BKR96] and [Fie06], see Corollary 4.8.

Another main result provides a tropical analogue of the Loewner-Whitney theorem Whi52, Loe55] and [FZ00, Theorem 12]. The classical theorem shows that any invertible totally nonnegative matrix is a product of nonnegative elementary Jacobi matrices.

Theorem B. We have

$$
\operatorname{val}\left(\mathrm{GL}_{n} \cap \mathrm{TN}\right)=\langle\text { tropical Jacobi elementary matrices }\rangle .
$$

This is part of Theorem 5.2 below. Here, $\mathrm{GL}_{n}$ denotes the set of invertible $n \times n$ matrices over $\mathbb{K}$, and $\langle\cdot\rangle$ denotes the multiplicative semigroup generated by a set of matrices (i.e., the set of finite products of matrices in this set). Tropical Jacobi elementary matrices are defined in a way analogous to the classical situation, see Section [5. The semigroup they generate was studied in [Niv14]: whereas classically, every nonsingular matrix can be factored in terms of elementary matrices, the same is not true in the tropical setting. Nevertheless, it was shown there that the set of $3 \times 3$ tropical matrices which admits such a factorization admits a combinatorial characterization. The present result shows that this characterization can be interpreted in terms of total nonnegativity, and provides some generalization to the $n \times n$ case.

A complete comparison of the classes of matrices studied in the present paper can be found in Theorem $\mathrm{C}$ and Tables 1 and 2 below.

The paper comprises several other results. In particular, Theorem 3.5. which holds for matrices over Puiseux series, and in particular, for matrices over the field of real numbers, is a linear algebra result which may be of independent interest, it shows that if the $2 \times 2$ minors of a matrix with positive entries are positive and "sufficiently away" from 0 , then, this matrix is totally positive. Propositions 6.5 and 6.3 characterize the valuations of the eigenvalues of a totally positive matrix with entries in $\mathbb{K}$, showing these are nothing but the valuations of the diagonal entries of the matrix. Corollary 7.9 provides a tropical analogue of the representation of totally nonnegative matrices as weight matrices of planar networks.

1.3. Related results and other approaches. As mentioned above, a first source of inspiration of the present work is the classical theory of totally positive matrices; Fallat and Johnson [FJ11 and Fomin and Zelevinsky [FZ00] gave recent accounts of this theory. We exploited a characterization of Monge matrices, obtained by Burkard, Klinz, Rudolf and Fiedler [BKR96, Fie06].

The notion of "positivity" has other incarnations in linear algebra. In particular, the tropical analogues of positive definite matrices have been studied by $\mathrm{Yu}$, who showed in Yu15, that the image by the valuation of the set of symmetric positive definite matrices over the field of Puiseux series is characterized by the nonnegativity of its principal $2 \times 2$ minors. More generally, the tropicalization of "generic" spectrahedra involves $2 \times 2$ minors [AGS16a. We show that a somehow analogous property, $\operatorname{val}(\mathrm{TP})=\mathrm{TN}_{2}^{\text {trop }}(\mathbb{R})$, is valid for totally positive matrices.

Another approach to total positivity arises by considering, following Postnikov [Pos06, the totally nonnegative (or positive) Grassmannian. The latter consists of the elements of the Grassmannian that have nonnegative (or positive) Plücker coordinates. A survey on totally nonnegative Grassmannian and 
a new approach to Grassmann polytopes via canonical bases was given by Lam in Lam14. Speyer and Williams studied in [SW05] the "tropical totally positive Grassmannian". The latter arises as the image by the valuation of the totally positive Grassmannian over the nonarchimedean field of Puiseux series with real coefficients. Whereas the classical Grassmanian can be realized as the image of the map which sends a full rank matrix to its (projective) Plücker coordinates, the same approach, transposed to the tropical setting, only yields an inner approximation of the tropical Grassmannian, as shown by Herrmann, Joswig and Speyer SS04 and Fink and Rincón [FR15. Similarly, the totally positive Grassmannian can be realized as an image of the set of totally positive matrices. We shall see in Section 7.1 that when transposed to the tropical setting, this approach leads only to a linear parametrization of a subset of the tropical totally positive Grassmannian by tropical totally nonnegative matrices.

\section{Preliminaries: NONARChimedeAn Amoebas of SEMialgebraiC SETS}

It is convenient to summarize here the main properties of valued fields which will be used. We refer the reader to [EP05] for background on valued fields.

We consider a field $\mathscr{K}$ equipped with a total order relation $\geqslant$. We denote by $\mathscr{K}_{\geqslant 0}:=\{f \in \mathscr{K} \mid$ $f \geqslant 0\}$ the set of nonnegative elements of $\mathscr{K}$ and by $\mathscr{K}_{>0}:=\{f \in \mathscr{K} \mid f>0\}$ the set of its positive elements. We also assume that $\mathscr{K}$ is equipped with a non-archimedean valuation, i.e., a map val : $\mathscr{K} \rightarrow \mathbb{R} \cup\{-\infty\}$ satisfying the following properties

$$
\operatorname{val}(f+g) \leqslant \max (\operatorname{val}(f), \operatorname{val}(g)), \quad \operatorname{val}(f g)=\operatorname{val}(f)+\operatorname{val}(g), \quad \operatorname{val}(f)=-\infty \Longleftrightarrow f=0 .
$$

The image of $\mathscr{K}^{*}:=\mathscr{K} \backslash\{0\}$ by the non-archimedean valuation is a subgroup of $(\mathbb{R},+)$, called the value group.

The field $\mathscr{K}$ possesses a subring, $\mathscr{O}:=\{f \in \mathscr{K}: \operatorname{val}(f) \leqslant 0\}$. We say that the valuation val is convex if it satisfies the following property: for every $f \in \mathscr{O}$ and every $g \in \mathscr{K}$ we have the implication

$$
0 \leqslant g \leqslant f \Longrightarrow g \in \mathscr{O} .
$$

This is equivalent to the following property: for all $f, g \in \mathscr{K}$,

$$
f, g \geqslant 0 \Longrightarrow \operatorname{val}(f+g)=\max (\operatorname{val}(f), \operatorname{val}(g)) .
$$

An ordered field is said to be real closed if the set of nonnegative elements is precisely the set of squares, and if every polynomial of odd degree with coefficients in this field has at least one root in the same field. A theorem of Tarski shows that the first order theory of real closed fields is complete, see Mar02, Coro. 3.3.16. This entails that a property expressed by a first order sentence in the language of ordered fields which is valid in a special real closed field, like $\mathbb{R}$, is valid in any real closed field. We shall make use of this property in the sequel.

If $\mathscr{K}$ is a real closed field, then we say that a subset $\mathcal{S} \subset \mathscr{K}^{n}$ is basic semialgebraic if it is of the form

$$
\mathcal{S}=\left\{\left(f_{1}, \ldots, f_{n}\right) \in \mathscr{K}^{n}: \forall i \in[p], P_{i}\left(f_{1}, \ldots, f_{n}\right)>0 \wedge \forall i \in[q] \backslash[p], P_{i}\left(f_{1}, \ldots, f_{n}\right)=0\right\},
$$

where $P_{1}, \ldots, P_{q}$ are multivariate polynomials with coefficients in $\mathscr{K}$, and $1 \leqslant q \leqslant p$. We say that $\mathcal{S}$ is semialgebraic if it is a finite union of basic semialgebraic sets.

Gelfand, Kapranov, and Zelevinsky introduced in GKZ94 the notion of amoeba of an algebraic variety $V$ included in $\left(\mathbb{C}^{*}\right)^{n}$, as the image of this variety by the map $\left(z_{i}\right) \mapsto\left(\log \left|z_{i}\right|\right)$. Amoebas have also been considered in the nonarchimedean setting; $\mathbb{C}$ is now replaced by a non-archimedean field, and the log-of-modulus map is replaced by the nonarchimedean valuation EKL06. In the present work, we will be interested by amoebas of subsets defined by inequalities as well as by equalities. This leads to the following notion.

Definition 2.1. If $\mathcal{S}$ is a semi-algebraic subset of $\mathscr{K}_{>0}^{n}$, where $\mathscr{K}$ is a real closed field equipped with a nonarchimedean valuation val, the amoeba of $\mathcal{S}$ is the set $\operatorname{val}(\mathcal{S}):=\left\{\left(\operatorname{val} f_{1}, \ldots, \operatorname{val} f_{n}\right) \mid\left(f_{1}, \ldots, f_{n}\right) \in\right.$ $\mathcal{S}\} \subset \mathbb{R}^{n}$.

Such amoebas have a polyhedral structure. Recall that a set $S \subset \mathbb{R}^{n}$ is basic semilinear if it is of the form

$$
S=\left\{\left(x_{1}, \ldots, x_{n}\right) \in \mathbb{R}^{n}: \forall i \in[p], \ell_{i}\left(x_{1}, \ldots, x_{n}\right)>h^{(i)} \wedge \forall i \in[q] \backslash[p], \ell_{i}\left(x_{1}, \ldots, x_{n}\right)=h^{(i)}\right\},
$$


where $\ell_{1}, \ldots, \ell_{q}$ are linear forms with integer coefficients, $h^{(1)}, \ldots, h^{(q)} \in \mathbb{R}$, and $1 \leqslant p \leqslant q$. We say that $S$ is semilinear if it is a finite union of basic semilinear sets.

The following result is derived in AGS16b as a corollary of a quantifier elimination result for valued fields of Denef [Den86] and Pas [Pas89]. A related result was proved by Alessandrini, in the setting of o-minimal geometry Ale13.

Theorem 2.2 (AGS16b, Theorem 4], see also Ale13, Theorem 3.11]). Let $\mathscr{K}$ be a real closed field equipped with a convex nonarchimedean valuation val with value group $\mathbb{R}$. Furthermore, suppose that the set $\mathcal{S} \subset \mathscr{K}_{>0}^{n}$ is semialgebraic. Then $\operatorname{val}(\mathcal{S})$ is a semilinear subset of $\mathbb{R}^{n}$.

It is also known that this semilinear subset is closed in the Euclidean topology AGS16b, Theorem 10].

In the sequel, we shall be interested in the amoebas of semialgebraic sets over a nonarchimedean field, especially, the set of totally positive matrices and the "totally positive part" of the Grassmanian. Hence, it may help to keep in mind Theorem 2.2. However, we emphasize that our main results do not rely on this theorem, but rather proceed by direct characterizations.

We now give examples of real closed nonarchimedean ordered fields with a convex valuation.

Example 2.3. A Hahn series is of the form

$$
\sum_{\lambda \in \Lambda} a_{\lambda} t^{\lambda}
$$

where $a_{\lambda} \in \mathbb{R} \backslash 0$ and $-\Lambda$ is a well ordered subset of $\mathbb{R}$, with the convention that the latter sum is zero if $\Lambda$ is empty. This field is denoted by $\left[\left[\mathbb{R}^{(\mathbb{R}, \leqslant)}\right]\right]$ in $[\mathrm{Rib92},(6.10)]$, where it is shown to be real closed.

The following smaller field is a popular choice in tropical geometry [Mar10.

Example 2.4. A generalized Puiseux series is a series of the form (2.4), where $\Lambda$ is either empty, or finite, or coincides with the set of elements of a sequence of real numbers decreasing to $-\infty$. This is precisely the field considered in the introduction, and denoted by $\mathbb{K}$ there. It follows for instance from a result of [Mar10] that this field is real closed. Indeed, the latter reference considers the field of formal generalized Puiseux series with complex coefficients. This field, which can be identified to the quadratic extension $\mathbb{K}[\sqrt{-1}$, is shown to be algebraically closed in Mar10. This implies that $\mathbb{K}$ is real closed. In the sequel, we shall often think of $\mathbb{K}$ as the subset of "real" elements of $\mathbb{K}[\sqrt{-1}]$, extending the classical terminology and notation for complex numbers, like "real part", "imaginary part", and "modulus", to $\mathbb{K}[\sqrt{-1}]$. E.g., the modulus of $c=a+(\sqrt{-1}) b$ with $a, b \in \mathbb{K}$ is $|c|=\sqrt{a^{2}+b^{2}} \in \mathbb{K}$.

Example 2.5. The field $\left[\left[\mathbb{R}^{(\mathbb{R}, \leqslant)}\right]\right]$ of Hahn series and the field $\mathbb{K}$ of generalized Puiseux series have subfields, consisting of series that are absolutely convergent for all sufficiently small positive values of $t$. We shall denote by $\left[\left[\mathbb{R}^{(\mathbb{R}, \leqslant)}\right]\right]_{\mathrm{cvg}}$ and $\mathbb{K}_{\mathrm{cvg}}$ these two fields, respectively. van den Dries and Speissegger showed in vdDS98, Corollary 9.2] that $\left[\left[\mathbb{R}^{(\mathbb{R}, \leqslant)}\right]\right]_{\mathrm{cvg}}$ is real closed; indeed, $\left[\left[\mathbb{R}^{(\mathbb{R}, \leqslant)}\right]\right]_{\mathrm{cvg}}$ is precisely the field of germs of functions definable in a certain o-minimal structure $\mathbb{R}_{\text {an* }}$. They also observed in Section 10.2 , ibid., that the same proofs apply to $\mathbb{K}_{\mathrm{cvg}}$, which entails in particular that this field is also real closed. We finally note that the field $\mathbb{K}_{\mathrm{cvg}}$ is isomorphic to the field of (absolutely convergent) generalized Dirichlet series considered by Hardy and Riesz [HR15]. Ordinary Dirichlet series are of the form $\sum_{n \geqslant 1} a_{n} n^{-s}$; they can be identified to series of the form (1.1), setting $\lambda_{n}=-\log n$, with the substitution $s:=\exp (t)$.

A map cs: $\mathbb{R} \rightarrow \mathscr{K}^{*}$ is called a cross-section if it is a multiplicative morphism such that val o cs is the identity map. For instance, if $\mathscr{K}$ is any of the ordered fields in Examples 2.3 2.5, the map $y \mapsto t^{y}$ is a cross section. We shall frequently use this cross section in the proofs which follow. More generally, every real closed valued field with a convex nonarchimedean valuation and value group $\mathbb{R}$ has a cross-section AGS16b, Lemma 3].

To keep our exposition as concrete as possible, we shall assume in the sequel that $\mathscr{K}=\mathbb{K}$ is the field of generalized Puiseux series considered in Example 2.4. Our results hold, without changes, if $\mathbb{K}$ is replaced by the field of Hahn series (Example 2.3), or by fields of absolutely convergent series like the ones of Example 2.5. More generally, we leave it to the reader to check that our arguments hold in any real closed field with a convex nonarchimedean valuation and value group $\mathbb{R}$, referring to [Ale13] and 
to AGS16b] for more details on the tropicalization of semialgebraic sets over ordered nonarchimedean fields, including (in AGS16b]) situations in which the value group is not necessarily $\mathbb{R}$.

\section{Characterizing tropical total POSITIVITy in terms of $2 \times 2$ Minors}

This section follows the notation and results in [FJ11, §3.1]. Total positivity of an $n \times m$ matrix may be verified by the positivity of its $n m$ initial minors GP95. We show that in the tropical setting, total nonnegativity can be checked by considering $n m 2 \times 2$ solid minors. We also show that if these minors are non- $\mathbb{O}$, then the matrix is uniquely determined by them. We shall also see that tropical total positivity implies some kind of diagonal dominance.

We shall need the following immediate fact.

Lemma 3.1. Let $\pi \in S_{n}$ be a permutation different from the identity permutation Id. There exists $i, j$ such that $i>j$ and $\pi(i)<\pi(j)$, called an inversion of $i, j$ in $\pi$. Moreover, we may choose $j=\pi(i)$.

We recall that a real matrix $A$ is a Monge matrix if and only if it satisfies the Monge property

$$
A_{i, j}+A_{i^{\prime}, j^{\prime}} \geqslant A_{i, j^{\prime}}+A_{i^{\prime}, j}, \quad \text { for all } i<i^{\prime} \text { and } j<j^{\prime} .
$$

A strict Monge matrix is obtained by requiring the above inequalities to be strict. (More precisely, the Monge property is defined by the reversed inequalities, whereas the definition above is the anti-Monge property. We omit the notion "anti" throughout.) Note that $\mathrm{TP}_{2}^{\text {trop }}$ and $\mathrm{TN}_{2}^{\text {trop }}(\mathbb{R})$ are by definition the sets of strict Monge matrices and respectively Monge matrices. The following observation is classical.

Lemma 3.2 ([BKR96]). A real matrix is a (strict) Monge matrix if and only if the (strict) relations (3.1) hold for consecutive values of $i, i^{\prime}$ and of $j, j^{\prime}$.

We shall also use the following easy observation, showing that the valuation restricted to the set of nonnegative (generalized) Puiseux series is an order preserving map:

$$
f, g \in \mathbb{K}_{\geqslant 0} \text { and } f \geqslant g \Longrightarrow \operatorname{val} f \geqslant \operatorname{val} g \text {. }
$$

We start by an elementary lemma.

Lemma 3.3. $\operatorname{val}\left(\mathrm{TN}_{2}\right)=\mathrm{TN}_{2}^{\text {trop }}$.

Proof. Suppose that $\boldsymbol{A}=\left(\boldsymbol{A}_{i j}\right) \in \mathrm{TN}_{2}$, and let $A_{i j}:=$ val $\boldsymbol{A}_{i j}$. By definition of $\mathrm{TN}_{2}$, for all $i<j$ and $k<l$, we have $\boldsymbol{A}_{i k} \boldsymbol{A}_{j l} \geqslant \boldsymbol{A}_{i l} \boldsymbol{A}_{j k}$, and by (3.2), $A_{i k}+A_{j l} \geqslant A_{i l}+A_{j k}$, which implies that $A \in \mathrm{TN}_{2}^{\text {trop }}$. Conversely, if $A \in \mathrm{TN}_{2}^{\text {trop }}$, we immediately check that the canonical lift $\boldsymbol{A}$ of $A, \boldsymbol{A}_{i j}:=t^{A_{i j}} \in \mathbb{K}$, belongs to TN.

We now show that the tropical totally nonnegative real matrices are precisely the Monge matrices.

Theorem 3.4. $\mathrm{TP}^{\text {trop }}=\mathrm{TP}_{2}^{\text {trop }}$ and $\mathrm{TN}^{\text {trop }}=\mathrm{TN}_{2}^{\text {trop }}$.

In the proof, and in the sequel, for all $I \subset[n]$ and $J \subset[m]$, we denote by $A_{I, J}$ the $I \times J$ submatrix of $A$.

Proof. By definition $\mathrm{TP}^{\text {trop }} \subset \mathrm{TP}_{2}^{\text {trop }}$ and $\mathrm{TN}^{\text {trop }} \subset \mathrm{TN}_{2}^{\text {trop }}$.

Assume $A \notin \mathrm{TN}^{\text {trop }}$. Therefore, for some $I$, $J$ s.t. $|I|=|J|>2$, all the permutations of maximum weight in $\operatorname{per}\left(A_{I, J}\right)$ are odd. Let $\pi$ be such an odd permutation, and denote by $M$ the $2 \times 2$ submatrix $A_{\{j, i\},\{\pi(i), \pi(j)\}}$, for some inversion of $i>j$ in $\pi$. Suppose that $\operatorname{per}(M)=A_{j, \pi(i)} \odot A_{i, \pi(j)} \geqslant$ $A_{i, \pi(i)} \odot A_{j, \pi(j)}$, and consider $\sigma:=\pi \circ(\pi(i) \pi(j))$, where $(k, l)$ denotes the transposition of indices $k, l$, and $\circ$ denotes the composition of permutations. Then, $\sigma$ is still of maximum weight in $\operatorname{per}\left(A_{I, J}\right)$, and it is even, contradicting the assumption. It follows that $\operatorname{per}(M)=A_{i, \pi(i)} \odot A_{j, \pi(j)}>A_{j, \pi(i)} \odot A_{i, \pi(j)}$, and $A \notin \mathrm{TN}_{2}^{\text {trop }}$.

Similarly, if $A \notin \mathrm{TP}^{\text {trop }}$, then there exists at least one odd permutation $\pi$ of maximum weight in $\operatorname{per}\left(A_{I, J}\right)$. If $\operatorname{per}(M)=A_{j, \pi(i)} \odot A_{i, \pi(j)}>A_{i, \pi(i)} \odot A_{j, \pi(j)}$, then the weight of the permutation $\pi \circ$ $(\pi(i) \pi(j))$ is strictly bigger than the maximal weight of $\pi$ in $\operatorname{per}\left(A_{I, J}\right)$. Thus $\operatorname{per}(M)=A_{i, \pi(i)} \odot$ $A_{j, \pi(j)} \geqslant A_{j, \pi(i)} \odot A_{i, \pi(j)}$, and therefore $A \notin \mathrm{TP}_{2}^{\text {trop }}$. Additionally, if $\operatorname{per}\left(A_{I, J}\right)=-\infty$, then $A$ has $-\infty$ entry, and therefore $A \notin \mathrm{TP}_{2}^{\text {trop }}$. 
It seems to be a general principle in tropical geometry that properties over the tropical semiring translate to weaker or approximate properties over fields, see e.g. AKNR13, AGS17 for applications of this principle to the localization of roots of polynomials. According to the same principle, we expect the characterization of tropical totally positive matrices in Theorem 3.4 to translate to a sufficient condition for the total positivity of matrices over a real field. We next give such a condition.

Given $C \geqslant 1$, we denote by $\mathrm{TN}_{2, C}$ the set of matrices $\boldsymbol{A}$ such that

$$
\boldsymbol{A}_{i, j} \boldsymbol{A}_{i^{\prime}, j^{\prime}} \geqslant C \boldsymbol{A}_{i, j^{\prime}} \boldsymbol{A}_{i^{\prime}, j}, \forall i<i^{\prime} \text { and } j<j^{\prime} .
$$

The set $\mathrm{TP}_{2, C}$ denotes the subset of $\mathrm{TN}_{2, C}$ obtained by requiring each of the above inequalities to be strict. Note that the Vandermonde matrix $\left(V_{i, j}\right)=\left(\lambda_{i}^{j-1}\right)$ with $0<\lambda_{1}<\cdots<\lambda_{n}$ is in $\mathrm{TP}_{2, C}$ as soon as $\lambda_{i+1} / \lambda_{i}>C$, for all $1 \leqslant i \leqslant n-1$, and therefore $\emptyset \neq \mathrm{TP}_{2, C} \subset \mathrm{TN}_{2, C}$ for every $C \geqslant 1$. The following theorem, which shows that

$$
\mathrm{TN}_{2, C} \subset \mathrm{TN} \subset \mathrm{TN}_{2}
$$

for some value of $C$ depending on $n, m$, may be thought of as an archimedean analogue of Theorem 3.4 Note that this theorem is valid in particular for matrices with entries in the field of real numbers.

Theorem 3.5. If $\boldsymbol{A} \in\left(\mathrm{TN}_{2, C}\right)^{n \times m}$ with $C \geqslant(\min (n, m)-1)^{2}$, then $\boldsymbol{A} \in \mathrm{TN}$. Similarly, under the same condition on $C$, if $\boldsymbol{A} \in\left(\mathrm{TP}_{2, C}\right)^{n \times m}$ then $\boldsymbol{A} \in \mathrm{TP}$.

The proof of this result relies on a series of auxiliary results.

Lemma 3.6. Let $\boldsymbol{A} \in\left(\mathrm{TN}_{2, C}\right)^{n \times n}$. Let $\gamma$ be any cyclic permutation of a subset $I$ of elements of $[n]$. Then,

$$
\prod_{j \in I} \boldsymbol{A}_{j, \gamma(j)} \leqslant \frac{1}{C^{|I|-1}} \prod_{j \in I} \boldsymbol{A}_{j, j}
$$

Moreover, the above inequality is strict as soon as $\boldsymbol{A} \in\left(\mathrm{TP}_{2, C}\right)^{n \times n}$.

Proof. We show that (3.3) holds for all cyclic permutations of a subset $I \subset[n]$ by induction on the number of elements $k$ of this subset. The base of the induction, $|I|=2$, holds by definition of $\operatorname{TN}_{2, C}^{n \times n}$.

Consider now a cycle $\gamma=\left(i_{1} \ldots i_{k}\right)$. A cycle must have an inversion, and we may assume without loss of generality that this inversion is on the indices $i_{k-1}, i_{k}$, so that $i_{k-1}<i_{k}$ and $i_{k}>i_{1}$. Considering the $\left\{i_{k-1}, i_{k}\right\} \times\left\{i_{1}, i_{k}\right\}$ submatrix of $\boldsymbol{A}$, we observe that

$$
\boldsymbol{A}_{i_{k-1} i_{k}} \boldsymbol{A}_{i_{k} i_{1}} \leqslant \frac{1}{C} \boldsymbol{A}_{i_{k-1} i_{1}} \boldsymbol{A}_{i_{k} i_{k}}
$$

It follows that

$$
\boldsymbol{A}_{i_{1}, i_{2}} \boldsymbol{A}_{i_{2}, i_{3}} \cdots \boldsymbol{A}_{i_{k-2}, i_{k-1}}\left(\boldsymbol{A}_{i_{k-1}, i_{k}} \boldsymbol{A}_{i_{k}, i_{1}}\right) \leqslant \frac{1}{C}\left(\boldsymbol{A}_{i_{1}, i_{2}} \boldsymbol{A}_{i_{2}, i_{3}} \ldots \boldsymbol{A}_{i_{k-1}, i_{1}}\right) \boldsymbol{A}_{i_{k} i_{k}}
$$

Applying the induction hypothesis to the cycle $\left(i_{1}, \ldots, i_{k-1}\right)$, we obtain (3.3).

The case of strict inequalities is obtained in the same way, using the fact that all entries of a matrix in $\mathrm{TP}_{2, C}$ are positive.

Consider a matrix $\boldsymbol{F} \in \mathbb{K}^{n \times n}$, and define the maximal cycle mean of $\boldsymbol{F}$,

$$
\rho_{\max }(\boldsymbol{F}):=\sup _{i_{1} \ldots i_{k}}\left|\boldsymbol{F}_{i_{1}, i_{2}} \ldots \boldsymbol{F}_{i_{k}, i_{1}}\right|^{\frac{1}{k}}
$$

where the maximum is taken over all sequences $i_{1}, \ldots, i_{k}$ of distinct elements of $\{1, \ldots, n\}$. The following result gives a sufficient condition for the determinant of a matrix to have the same sign as the determinant of its diagonal.

Theorem 3.7. Suppose that $\boldsymbol{A} \in \mathbb{K}^{n \times n}$ can be written as

$$
\boldsymbol{A}=\boldsymbol{D}+\boldsymbol{B}
$$

where $\boldsymbol{D}$ is a diagonal matrix with non-zero diagonal entries, and $\boldsymbol{B}$ has zero diagonal entries. If

$$
\rho_{\max }\left(\boldsymbol{D}^{-1} \boldsymbol{B}\right)<1 /(n-1),
$$


then

$$
(\operatorname{det} \boldsymbol{D})^{-1} \operatorname{det} \boldsymbol{A} \geqslant\left(1-(n-1) \rho_{\max }\left(\boldsymbol{D}^{-1} \boldsymbol{B}\right)\right)^{n}>0
$$

in particular, $\operatorname{det} \boldsymbol{D} \operatorname{det} \boldsymbol{A}>0$. Moreover, if we only have a weak inequality in (3.4), then $\operatorname{det} \boldsymbol{D} \operatorname{det} \boldsymbol{A} \geqslant$ 0 .

Proof. A matrix $\boldsymbol{F} \in \mathbb{K}^{n \times n}$ has $n$ eigenvalues in $\mathbb{K}[\sqrt{-1}]$, counted with multiplicities, and we denote by $\rho(\boldsymbol{F})$ the spectral radius of $\boldsymbol{F}$, i.e., the maximum of the moduli of these eigenvalues. We denote by $\operatorname{pat}(\boldsymbol{F})$ the Boolean pattern of the matrix $\boldsymbol{F}$, so that the $(i, j)$-entry of pat $(\boldsymbol{F})$ is equal to 1 if $\boldsymbol{F}_{i j} \neq 0$, and to 0 otherwise. Let us recall the following inequality of Friedland [Fri86], relating the maximal cycle mean with the spectral radius:

$$
\rho(\boldsymbol{F}) \leqslant \rho_{\max }(\boldsymbol{F}) \rho(\operatorname{pat}(\boldsymbol{F})) .
$$

Indeed, this inequality is established in Fri86 for matrices with entries in $\mathbb{R}$. This property can be expressed by a first order sentence in the language of ordered fields, and so, by Tarski's completeness theorem mentioned in Section 2, it also holds for matrices with entries in $\mathbb{K}$.

Consider $\boldsymbol{F}:=\boldsymbol{D}^{-1} \boldsymbol{B}$, so that $\boldsymbol{D}^{-1} \boldsymbol{A}=\boldsymbol{I}+\boldsymbol{F}$. Since $\boldsymbol{B}_{i i}=0$, the matrix $\boldsymbol{P}:=\operatorname{pat}(\boldsymbol{F})$, which as $0 / 1$ entries, has at most $n-1$ entries equal to 1 in each row. Hence, $\rho(\boldsymbol{P}) \leqslant \max _{i} \sum_{j}\left|\boldsymbol{P}_{i j}\right| \leqslant n-1$. Let $\boldsymbol{\lambda}_{1}, \ldots, \boldsymbol{\lambda}_{n} \in \mathbb{K}[\sqrt{-1}]$ denote the eigenvalues of $\boldsymbol{F}$. We deduce from (3.6) that if $\rho_{\max }(\boldsymbol{F})<1 /(n-1)$, then $\left|\boldsymbol{\lambda}_{i}\right| \leqslant(n-1) \rho_{\max }(\boldsymbol{F})<1$, for all $1 \leqslant i \leqslant n$. Observe that

$$
(\operatorname{det} \boldsymbol{D})^{-1} \operatorname{det} \boldsymbol{A}=\operatorname{det}\left(\boldsymbol{D}^{-1} \boldsymbol{A}\right)=\prod_{1 \leqslant i \leqslant n}\left(1+\boldsymbol{\lambda}_{i}\right)
$$

If $\boldsymbol{\lambda}_{i}$ is real, we have $1+\boldsymbol{\lambda}_{i} \geqslant 1-\left|\boldsymbol{\lambda}_{i}\right| \geqslant 1-(n-1) \rho_{\max }(\boldsymbol{F})>0$. Otherwise, we note that both $\boldsymbol{\lambda}_{i}$ and its conjugate $\overline{\boldsymbol{\lambda}}_{i}$ are eigenvalues of $\boldsymbol{F}$, and observe that $\left(1+\boldsymbol{\lambda}_{i}\right)\left(1+\overline{\boldsymbol{\lambda}}_{i}\right) \geqslant\left(1-\left|\boldsymbol{\lambda}_{i}\right|\right)^{2} \geqslant$ $\left(1-(n-1) \rho_{\max }(\boldsymbol{F})\right)^{2}>0$. Therefore, regrouping the eigenvalues by conjugate pairs, we deduce that

$$
\prod_{1 \leqslant i \leqslant n}\left(1+\boldsymbol{\lambda}_{i}\right) \geqslant\left(1-(n-1) \rho_{\max }(\boldsymbol{F})\right)^{n}>0,
$$

which shows 3.5

If only the weak inequality holds in (3.4), we can still conclude that the weak inequality holds in (3.7).

Proof of Theorem 3.5. We need to show that every minor of $\boldsymbol{A}$ is nonnegative (or positive). Hence, possibly after replacing $\boldsymbol{A}$ by a submatrix, we may assume that $n=m$. We also assume without loss of generality that $\boldsymbol{A}_{i, i}=1$ for all $i \in[n]$. We set $\boldsymbol{A}=\boldsymbol{B}+\boldsymbol{I}$, where $\boldsymbol{I}$ is the identity matrix, and $\boldsymbol{B}$ is the off-diagonal part of $\boldsymbol{A}$ meaning that $\boldsymbol{B}_{i, j}=\boldsymbol{A}_{i j}$ if $i \neq j$ and $\boldsymbol{B}_{i, i}=0$. Lemma 3.6 yields the following bound on the maximal cycle mean of $\boldsymbol{B}$,

$$
\rho_{\max }(\boldsymbol{B}) \leqslant \max _{2 \leqslant k \leqslant n} \frac{1}{\left(C^{k-1}\right)^{\frac{1}{k}}}=\frac{1}{C^{\frac{1}{2}}} .
$$

If $C \geqslant(n-1)^{2}, \rho_{\max }(\boldsymbol{B}) \leqslant 1 /(n-1)$, and we deduce from Theorem 3.7 that det $\boldsymbol{A} \geqslant 0$. Similarly, if $\boldsymbol{A} \in \mathrm{TP}_{2, C}$, we deduce along the same lines that $\operatorname{det}(\boldsymbol{A})>0$.

Remark 3.8. The statement of Theorem 3.5 is inspired by an analogous result for symmetric matrices which has appeared in AGS16a. It is shown there that if $\boldsymbol{A}$ is an $n \times n$ symmetric matrix with nonnegative diagonal entries, such that $\boldsymbol{A}_{i, i} \boldsymbol{A}_{j, j} \geqslant(n-1)^{2} \boldsymbol{A}_{i, j}^{2}$ for all $i<j$, then, $\boldsymbol{A}$ is positive semidefinite.

We now introduce the tropical notion of diagonal dominance, following the terminology of But03, $\S 3]$ : a matrix $A \in \mathbb{R}_{\max }^{n \times n}$ is tropical diagonally dominant if $\operatorname{per}(A)=\sum_{i \in[n]} A_{i, i}$, and it is tropical strictly diagonally dominant if $\operatorname{per}(A)>\sum_{i \in[n]} A_{i, \sigma(i)}$ holds for all permutation $\sigma$ different from the identity. We denote by DD ${ }^{\text {trop }}$ the set of matrices such that every submatrix is tropical diagonally dominant, and by SDD ${ }^{\text {trop }}$ the set of matrices such that every submatrix is tropical strictly diagonally dominant.

Proposition 3.9. $\mathrm{TN}^{\text {trop }} \subset \mathrm{DD}^{\text {trop }}$ and $\mathrm{TP}^{\text {trop }} \subset \mathrm{SDD}^{\text {trop }}$. 
Proof. We show that if $A \in \mathrm{TN}^{\text {trop }}$ is $n \times n$, then the identity permutation attains the permanent of $A$. We have trivially $\mathrm{TN}^{\text {trop }} \subset \mathrm{TN}_{2}^{\text {trop }}$, and we observed in the proof of Lemma 3.3 that if $A \in \mathrm{TN}_{2}^{\text {trop }}$, then the canonical lift $\left(t^{A_{i j}}\right)$ belongs to $\mathrm{TN}_{2}(\mathbb{K})$. The latter set coincides with $\mathrm{TN}_{2, C}(\mathbb{K})$, for $C=1$. It follows from Lemma 3.6 that for all cycle $\gamma$ with set of elements $I$,

$$
\prod_{j \in I} t^{A_{j, \gamma(j)}} \leqslant \prod_{j \in I} t^{A_{j, j}}
$$

Thus the weight of any permutation of $A$ is dominated by the weight of the identity permutation.

The case in which $A \in \mathrm{TP}^{\text {trop }}$ follows by noting that the inequalities (3.9) are strict.

It follows from Lemma 3.2 that a real matrix $A$ is in $\mathrm{TN}^{\text {trop }}(\mathbb{R})$ (resp. in $\mathrm{TP}^{\text {trop }}$ ) if and only if its $2 \times 2$ solid minors are tropically nonnegative (resp. tropically positive). Therefore, for an $n \times m$ matrix to be tropical totally nonnegative (resp. positive) with real entries, it suffices that the following $n m$ conditions hold: each of the entries of the first row and first column is real and all the $2 \times 2$ solid submatrices satisfy the Monge property (resp. strict Monge property). Note that the conclusion of Lemma 3.2 does not carry over to the situation in which $A$ has $\mathbb{O}$ entries, as seen for instance by $A=\left(\begin{array}{lll}2 & -\infty & 2 \\ 2 & -\infty & 0\end{array}\right)$.

Classically, there are $n m$ sufficient conditions for an $n \times m$ matrix to be totally positive, given by the positivity of its initial minors (solid and bordering either the left or the top edge of the matrix). In the following proposition, we provide the tropical analogue of this property.

Proposition 3.10. Let $A \in \mathbb{R}^{n \times m}$. The following are equivalent:

(1) The solid $2 \times 2$ tropical minors of $A$ are tropically positive,

(2) The tropical initial minors of $A$ are tropically positive,

(3) $A \in \mathrm{TP}^{\text {trop }}$.

Proof. We already proved that (3) and (10) are equivalent (Lemma 3.2). Moreover, (3) trivially implies (2).

We show that (2) implies (11). If the tropical initial minors of $A$ are tropically positive, then the initial minors of any lift of $A$ are positive. Thus, any lift $\boldsymbol{A}$ of $A$ is in TP, and in particular, the canonical lift $\boldsymbol{A}:=\left(t^{A_{i j}}\right)$ is in TP. It follows that every $2 \times 2$ minor of $\boldsymbol{A}$ is positive, which implies, since the lift is canonical, that every $2 \times 2$ minor of $A$ is tropically positive.

As in the classical case, $A$ is not necessarily in $\mathrm{TN}^{\text {trop }}$, if we allow (sign-)singular initial minors. See for instance the matrix $\left(\begin{array}{lll}1 & 1 & 1 \\ 1 & 1 & 3 \\ 2 & 2 & 1\end{array}\right)$, which has classically and tropically nonnegative initial minors, however its $2 \times 2$ bottom-right minor is classically and tropically negative.

Proposition 3.11. A tropical totally nonnegative real matrix is uniquely determined by the values of its $2 \times 2$ solid minors and by the entries on its first row and first column.

Proof. Denote by $M_{i, j}$ the permanent of the $2 \times 2$ submatrix of consecutive rows $\{i, i+1\}$ and consecutive columns $\{j, j+1\}$, of the matrix $A \in \mathrm{TN}^{\text {trop }}$. We have $A_{i-1, j-1} \odot A_{i, j}=M_{i-1, j-1}$, or $A_{i, j}=A_{i-1, j-1}^{\odot-1} \odot M_{i-1, j-1}$. This implies, after an immediate induction, that $A$ is well determined by the value of the $M_{i j}$, and by its first row and column.

\section{Double EChelon AND STAIrCASE Forms}

In this section, we describe the "shape" of tropical totally nonnegative matrices. We first consider the $\mathbb{O} /$ non- $\mathbb{O}$ pattern, showing that, as in the classical case (Fal99, FJ11, §1.6], the tropical matrices which have non- $\mathbb{0}$ rows and columns, have a double echelon form. Next, we characterize the set of tropical totally nonnegative matrices with finite entries. The latter constitute a polyhedron, which coincides with the set of (anti)-Monge matrices. Then, we deduce from a known characterization of Monge matrices BKR96, § 2], Fie06] that every tropical totally nonnegative real matrix is amenable to a certain "staircase form" by diagonal scaling. 
4.1. Double echelon form. Recall that a Boolean matrix is a matrix all of whose entries are 0 and 1 , and that the Boolean pattern of a matrix $A$ over a field, already considered in the proof of Theorem 3.7 is the Boolean matrix $B$ such that $B_{i, j}=1$ if $A_{i, j} \neq 0$, and 0 otherwise.

Definition 4.1. An $n \times m$ Boolean matrix has a double echelon pattern if

(1) Every row is in one of the following forms

(a) $(1, \ldots, 1)$,

(b) $(1, \ldots, 1,0, \ldots, 0)$,

(c) $(0, \ldots, 0,1, \ldots, 1,0, \ldots, 0)$,

(d) $(0, \ldots, 0,1, \ldots, 1)$,

(2) The first and last nonzero entries in row $i$ appear not to the left of the first and last nonzero entries in row $i-1$ respectively, $\forall i=2, \ldots, n$.

A matrix over $\mathbb{R}$ or $\mathbb{K}$ is in double echelon form if it has a double echelon Boolean pattern. A matrix over $\mathbb{R}_{\max }$ is in double echelon form if its lifts have a double echelon Boolean pattern.

Example 4.2. Every lift of

$$
E=\left(\begin{array}{ccccc}
1 & 1 & 3 & -\infty & -\infty \\
-\infty & 2 & 1 & 2 & -\infty \\
-\infty & 1 & 1 & 1 & 3 \\
-\infty & -\infty & 1 & 1 & 1
\end{array}\right)
$$

have the double echelon pattern

$$
\left(\begin{array}{lllll}
1 & 1 & 1 & 0 & 0 \\
0 & 1 & 1 & 1 & 0 \\
0 & 1 & 1 & 1 & 1 \\
0 & 0 & 1 & 1 & 1
\end{array}\right)
$$

Proposition 4.3. A tropical totally nonnegative matrix $A$ with no $\mathbb{O}$ row or column is in double echelon form.

Proof. Suppose first, by contradiction, that $A$ does not satisfy condition (1). Then there exists $i \in[n]$ such that row $A_{i}$ does not have a Boolean pattern as in (a)-(d). That is, there exist $t<k<\ell$ such that $A_{i, t} \neq \mathbb{O}, A_{i, k}=\mathbb{O}$ and $A_{i, \ell} \neq \mathbb{O}$. Since $A$ does not include zero columns, there exists row $j$ such that $A_{j, k} \neq \mathbb{O}$, and therefore the $\{i, j\} \times\{k, \ell\}$ minor is tropically negative.

Suppose now that $A$ satisfies condition (11) but not condition (2). Then there exists $i$ such that the first (or resp. last) non- $\mathbb{O}$ entry $A_{i, j}$ in row $i$ appears to the left of the first (or resp. last) non- $\mathbb{D}$ entry $A_{i-1, k}$ in row $i-1$. As a result, the $\{i-1, i\} \times\{j, k\}$ minor is tropically negative.

The following corollary is a straightforward consequence of Proposition 4.3 , since a matrix with no $\mathbb{0}$ maximal solid minor cannot have a $\mathbb{D}$-row and a no $\mathbb{D}$-column.

Corollary 4.4. If all the maximal solid minors of $A \in\left(\mathrm{TN}^{\text {trop }}\right)^{n \times m}$ are non- $\mathbb{0}$, then $A$ is in double echelon form.

4.2. Staircase matrices. For all $(i, j) \in([n] \backslash\{1\}) \times(j \in[m] \backslash\{1\})$, we define the elementary staircase matrix $L^{(i, j)} \in \mathbb{R}^{n \times m}$ as follows:

$$
L_{t, s}^{(i, j)}=\left\{\begin{array}{ll}
1 & \text { if }(t, s) \in[n] \backslash[i-1] \times[m] \backslash[j-1] \\
0 & \text { otherwise }
\end{array} .\right.
$$

For instance, for $m=n=3$,

$$
L^{(2,3)}=\left(\begin{array}{ccc}
0 & 0 & 0 \\
0 & 0 & 1 \\
0 & 0 & 1
\end{array}\right)
$$

A matrix $A \in \mathbb{R}^{n \times m}$ is a staircase matrix if it can be written as a nonnegative linear combination of elementary staircases matrices. We shall use the following characterization which was shown in [Fie06]; earlier characterizations of the same nature appeared in [BKR96, Lemma 2.1]. 
Theorem 4.5 ([Fie06, Theorem 3.3]). A matrix $A \in \mathbb{R}^{n \times m}$ is a Monge matrix if and only if there is a staircase matrix $S \in \mathbb{R}^{n \times m}$ and two vectors $u \in \mathbb{R}^{n}$ and $v \in \mathbb{R}^{m}$ such that

$$
A_{i j}=S_{i j}+u_{i}+v_{j}
$$

The following result follows readily by combining Theorem 3.4, showing that the matrices $\operatorname{TN}^{\text {trop }}(\mathbb{R})$ are precisely the Monge matrices, with Theorem 4.5.

Theorem 4.6. A matrix $A$ is in $\mathrm{TN}^{\text {trop }}(\mathbb{R})$ if and only if there exist tropical diagonal matrices $D, D^{\prime}$ such that $D \odot A \odot D^{\prime}$ is a staircase matrix.

Example 4.7. The following factorization illustrates this result:

$$
\begin{aligned}
\left(\begin{array}{ccc}
0 & -\infty & -\infty \\
-\infty & 1 & -\infty \\
-\infty & -\infty & 2
\end{array}\right) \odot \overbrace{\left(\begin{array}{ccc}
1 & 0 & -1 \\
0 & 1 & 0 \\
-1 & 0 & 1
\end{array}\right)}^{\text {in } \operatorname{TN}^{\text {trop }}(\mathbb{R})} \odot\left(\begin{array}{ccc}
-1 & -\infty & -\infty \\
-\infty & 0 & -\infty \\
-\infty & -\infty & 1
\end{array}\right) & =\left(\begin{array}{lll}
0 & 0 & 0 \\
0 & 2 & 2 \\
0 & 2 & 4
\end{array}\right) \\
& =2 L^{(2,2)}+2 L^{(3,3)}
\end{aligned}
$$

Theorem 4.6 provides a polyhedral characterization of $\mathrm{TN}^{\text {trop }}(\mathbb{R})$.

Corollary 4.8. The set $\mathrm{TN}^{\text {trop }}(\mathbb{R})$, thought of as a subset of $\mathbb{R}^{n \times m}$, is a polyhedron which can be written as the Minkowski sum $\mathrm{V}+\mathrm{S}$, where

$$
\mathrm{V}=\left\{\left(d_{i}+d_{j}^{\prime}\right)_{i \in[n], j \in[m]}, d \in \mathbb{R}^{n}, d^{\prime} \in \mathbb{R}^{m}\right\}
$$

is the $n+m-1$ dimensional lineality space of this polyhedron, and $\mathbf{S}$ is the set of staircase matrices, which is a simplicial cone of dimension $(n-1)(m-1)$ with extreme rays generated by the elementary staircase matrices $L^{(i, j)}$, for $(i, j) \in([n] \backslash\{1\}) \times(j \in[m] \backslash\{1\})$.

Proof. The decomposition of Theorem 4.6 shows that $\mathrm{TN}^{\operatorname{trop}}(\mathbb{R})=\mathrm{V}+\mathrm{S}$. Since $\mathrm{S}$ contains no affine line, $\mathrm{V}$ must coincide with the lineality space of $\mathrm{TN}^{\text {trop }}(\mathbb{R})$. It also follows from Theorem 4.6 that $\mathrm{S}$ is precisely the set of Monge matrices with a zero first row and a zero first column. Each matrix in this set can be written in a unique way as a positive linear combination of elementary staircase matrices, indeed,

$$
\begin{aligned}
A=\sum_{i=3}^{n} \sum_{j=3}^{m}\left(A_{i, j}+A_{i-1, j-1}-A_{i-1, j}-A_{i, j-1}\right) L^{(i, j)}+ & \\
& \sum_{j=3}^{m}\left(A_{2, j}-A_{2, j-1}\right) L^{(2, j)}+\sum_{i=3}^{n}\left(A_{i, 2}-A_{i-1,2}\right) L^{(i, 2)}+A_{2,2}^{(2,2)} L^{(2,2)}
\end{aligned}
$$

This implies that $\mathrm{S}$ is the $(n-1)(m-1)$-dimensional simplicial cone generated by the matrices $L^{(i, j)}$, $(i, j) \in([n] \backslash\{1\}) \times([m] \backslash\{1\})$. Observe that the dimensions of $\mathbf{S}$ and $\mathbf{V}$ sum to $\mathrm{nm}$, in accordance with $\mathrm{TN}^{\text {trop }}(\mathbb{R})$ being full dimensional (it has non empty interior).

Remark 4.9. Dually, the collection of inequalities

$$
A_{i, j}+A_{i+1, j+1} \geqslant A_{i, j+1}+A_{i+1, j},
$$

for $1 \leqslant i \leqslant n-1$ and $1 \leqslant j \leqslant m-1$ define $\operatorname{TN}^{\text {trop }}(\mathbb{R})$ (by Lemma 3.2). There are $(n-1)(m-1)$ inequalities of this kind. This collection of inequalities is minimal. Otherwise, we would eliminate some of these inequalities, and end up with a representation of the set of staircase matrices, identified to a polyhedral cone of $\mathbb{R}^{(n-1) \times(m-1)}$, by fewer than $(n-1)(m-1)$ inequalities. This is absurd, since it follows from Corollary 4.8 that this polyhedral cone is simplicial of dimension $(n-1)(m-1)$, so its number of facets, which coincides with the minimal cardinality of a defining set of inequalities, is also $(n-1)(m-1)$. 


\section{MATRIX FACTORIZATION AND LifTS OF TP ${ }^{\text {trop }}$ AND TN ${ }^{\text {trop }}$}

In this section, we relate the tropical of matrix classes with the image by the valuation of the corresponding matrix classes over a nonarchimedean field. In particular, we find a tropical analogue to the Loewner-Whitney theorem, which appeared in Loewner's work Loe55, being deduced there from a result of Whitney Whi52. This theorem shows that any invertible totally nonnegative matrix is a product of nonnegative elementary Jacobi matrices (see for instance [FZ00, Theorem 12]).

Definition 5.1. An (elementary) Jacobi matrix is an invertible matrix that differs from the identity matrix in at most one entry located either on the main diagonal or immediately above or below it. Analogously, an (elementary) tropical Jacobi matrix differs from the tropical identity matrix in at most one entry, which must be finite and located either on the main diagonal or immediately above or below it.

This definition follows the one of notion of [FZ00], and corresponds to the $L U$ factorization in [FJ11].

Theorem 5.2. Over $\mathbb{R}_{\max }^{n \times m}$ we have

$$
\mathrm{TP}^{\text {trop }} \varsubsetneqq \operatorname{val}(\mathrm{TP}) \varsubsetneqq \operatorname{val}(\mathrm{TN}) \subset \mathrm{TN}^{\text {trop }}, \quad \forall n, m
$$

and when $n=m$ we have

$$
\operatorname{val}\left(\mathrm{TP}^{n \times n}\right) \varsubsetneqq \operatorname{val}\left(\mathrm{GL}_{n} \cap \mathrm{TN}\right)=\langle\text { tropical Jacobi matrices }\rangle \varsubsetneqq \operatorname{val}\left(\mathrm{TN}^{n \times n}\right),
$$

where $\langle\cdot\rangle$ denotes the semigroup generated by a set of matrices.

Proof. We consider the canonical lift $\boldsymbol{A}$ of $A \in \mathrm{TP}^{\text {trop }}$, and note that val : $\mathbb{K}_{\geqslant 0} \rightarrow \mathbb{R}_{\max }$ is a morphism of semifields. For a $d \times d$ submatrix $M$ in $A$, indices naturally ordered, with the corresponding submatrix $\boldsymbol{M}=\left(t^{M_{i, j}}\right)$ in $\boldsymbol{A}$, we have

$$
\operatorname{det}(\boldsymbol{M})=\sum_{\sigma \in S_{d}} \operatorname{sgn}(\sigma) \prod_{i \in[d]} t^{M_{i, \sigma(i)}}=\sum_{\sigma \in S_{d}} \operatorname{sgn}(\sigma) t^{\sum_{i \in[d]} M_{i, \sigma(i)}} .
$$

Since the permutations of maximal weight in $\operatorname{per}(M)$ are even, the monomials with maximal valuation in the latter expansion have a positive sign, and so $\operatorname{det}(\boldsymbol{M}) \in \mathbb{K}_{>0}$, which shows that $\operatorname{TP}^{\text {trop }} \subset \operatorname{val}(\mathrm{TP})$. Note also in passing that the same proof shows that any lift $\boldsymbol{A}$ of $A$ is totally positive.

The first inclusion in (5.1) is strict since

$$
\boldsymbol{A}=\left(\begin{array}{cc}
2 t & t \\
1 & 1
\end{array}\right) \in \mathrm{TP} \quad \text { but } \operatorname{val}(\boldsymbol{A})=\left(\begin{array}{cc}
1 & 1 \\
0 & 0
\end{array}\right) \notin \mathrm{TP}^{\text {trop }}
$$

The second inclusion in (5.1), as well as the inclusions in (5.2), are trivial whereas the third inclusion in (5.1) follows from Lemma 3.3 and Theorem 3.4

The inclusions in (5.2), and the second inclusion in (5.1), are strict since

$$
\operatorname{val}\left(\begin{array}{ll}
1 & 0 \\
0 & 1
\end{array}\right) \in \operatorname{val}\left(\mathrm{GL}_{2} \cap \mathrm{TN}\right) \backslash \operatorname{val}(\mathrm{TP}) \text { and } \operatorname{val}\left(\begin{array}{ll}
1 & 0 \\
0 & 0
\end{array}\right) \in \operatorname{val}(\mathrm{TN}) \backslash \operatorname{val}\left(\mathrm{GL}_{2} \cap \mathrm{TN}\right) \text {. }
$$

The Loewner-Whitney theorem shows that $\mathrm{GL}_{n} \cap \mathrm{TN}=\langle$ nonnegative Jacobi matrices〉. Observe that the valuation sends the nonnegative Jacobi matrices with entries in $\mathbb{K}$ to the tropical Jacobi matrices. Since val : $\mathbb{K}_{\geqslant 0} \rightarrow \mathbb{R}_{\max }$ is a morphism of semifields, we deduce that the image by the valuation of the semigroup 〈nonnegative Jacobi matrices〉 is included in the semigroup 〈tropical Jacobi matrices〉. Conversely, val〈nonnegative Jacobi matrices〉 $\supset$ 〈tropical Jacobi matrices〉 since every tropical Jacobi matrix $J$ can be trivially lifted to a Jacobi matrix $\boldsymbol{J}$ over $\mathbb{K}$. Thus, if $A=J_{1} \odot \cdots \odot J_{k}$, then $\boldsymbol{A}$ such that $\operatorname{val}(\boldsymbol{A})=A$ may be defined by $\boldsymbol{J}_{1} \cdots \boldsymbol{J}_{k}$, where $\operatorname{val}\left(\boldsymbol{J}_{i}\right)=J_{i} \forall i$.

Theorem 5.3. A matrix $A$ is in $\mathrm{TP}^{\text {trop }}$ if and only if every lift of $A$ is in TP.

Proof. We already showed in the initial part of the proof of Theorem 5.2 that if $A$ is in TP ${ }^{\text {trop }}$, any lift of $A$ is in TP.

We now show the "if" part of the statement. We assume $A \notin \mathrm{TP}^{\text {trop }}$ and find a lift not in TP. Since, by Theorem 3.4. TP ${ }^{\text {trop }}=\mathrm{TP}_{2}^{\text {trop }}$, we deduce that either $A$ has an infinite entry, or that $A$ has a $2 \times 2$ submatrix, say its $\left\{i_{1}, i_{2}\right\} \times\left\{j_{1}, j_{2}\right\}$ submatrix, with a tropically nonpositive determinant. In the former case, every lift of $A$ has a 0 entry, and so no lift of $A$ can be totally positive. In the 
latter case, we have $A_{i_{1}, j_{2}}+A_{i_{2}, j_{1}} \geqslant A_{i_{1}, j_{1}}+A_{i_{2}, j_{2}}>-\infty$. Choose now any lift $\boldsymbol{A}$ of $A$ of the form $\boldsymbol{A}_{i, j}=b_{i, j} t^{A_{i, j}}$, with $b_{i, j}>1$ for $(i, j) \in\left\{\left(i_{1}, j_{2}\right),\left(i_{2}, j_{1}\right)\right\}$ and $b_{i, j}=1$ otherwise. It is immediate that $\boldsymbol{A}_{i_{1}, j_{2}} \boldsymbol{A}_{i_{2}, j_{1}}>\boldsymbol{A}_{i_{1}, j_{1}} \boldsymbol{A}_{i_{2}, j_{2}}$, showing that $\boldsymbol{A}$ is not totally positive.

An inspection of the proof above suggests the following more general construction. It will be convenient to denote by $*$ the Hadamard product (i.e., entrywise product) of two matrices. Let $\boldsymbol{B} \in \mathbb{K}^{n \times m}, A \in \mathbb{R}_{\max }^{n \times m}$, consider the canonical lift $t^{A}:=\left(t^{A_{i j}}\right)$, together with the Hadamard product $\boldsymbol{A}:=\boldsymbol{B} * t^{A}$, i.e.,

$$
\boldsymbol{A}_{i, j}:=\boldsymbol{B}_{i j} t^{A_{i j}}
$$

Observe that if $\boldsymbol{B} \in \mathbb{K}_{>0}^{n \times m}$ with val $\boldsymbol{B}_{i j}=0$ for all $i, j$, which is the case in particular if $\boldsymbol{B} \in \mathbb{R}_{>0}^{n \times m}$, then, $\boldsymbol{A}$ is a lift of $A$.

One may ask whether for $\boldsymbol{B} \in \mathrm{TN}^{n \times m}$, and $A \in\left(\mathrm{TN}^{\text {trop }}\right)^{n \times m}$, the matrix obtained by the Hadamard product construction (5.3) is in $\mathrm{TN}(\mathbb{K})$. The example of

$$
A=\left(\begin{array}{ccc}
0 & 0 & -\infty \\
0 & 0 & 0 \\
-\infty & 0 & 0
\end{array}\right) \in \mathrm{TN}^{\text {trop }} \text { and } \boldsymbol{B}=\left(\begin{array}{ccc}
1 & 1 & 1 \\
1 & 1 & 1 \\
1 & 1 & 1
\end{array}\right) \in \mathrm{TN}
$$

leading to $\operatorname{det} \boldsymbol{A}=-1$, shows that this is not necessarily true. The following result shows, however, that the conclusion becomes true if $\boldsymbol{B} \in \mathrm{TN}_{2, C}(\mathbb{K})$ for a suitable constant $C$. This will also provide a $\mathrm{TN}(\mathbb{K})$ lift for every $\mathrm{TN}^{\text {trop }}$ matrix.

Theorem 5.4. Let $A \in\left(\mathrm{TN}^{\text {trop }}\right)^{n \times m}$. For $\boldsymbol{B} \in \mathrm{TN}_{2,(n-1)^{2}}(\mathbb{K})$, the Hadamard product matrix $\boldsymbol{A} \in$ $\mathbb{K}^{n \times m}$ defined in (5.3) belongs to $\mathrm{TN}(\mathbb{K})$. Moreover, if $\operatorname{per}(A) \neq \mathbb{0}$ and $n=m$, then $\boldsymbol{A} \in \mathrm{GL}_{n} \cap \mathrm{TN}(\mathbb{K})$. If, in addition, $A \in \mathrm{TN}^{\text {trop }}(\mathbb{R})$, then $\boldsymbol{A} \in \mathrm{TP}(\mathbb{K})$.

To show this theorem, we make the following immediate observation.

Lemma 5.5. Given $C_{1}, C_{2} \geqslant 1$, if $M_{1} \in \mathrm{TN}_{2, C_{1}}$ and $M_{2} \in \mathrm{TN}_{2, C_{2}}$, then the Hadamard product $M_{1} * M_{2}$ is in $\mathrm{TN}_{2, C_{1} C_{2}}$.

Proof of Theorem 5.4. By Lemma 5.5. since $A \in \mathrm{TN}^{\text {trop }} \subset \mathrm{TN}_{2}^{\text {trop }}$, it is immediate that the canonical lift of $A$ is in $\mathrm{TN}_{2}(\mathbb{K})=\mathrm{TN}_{2,1}(\mathbb{K})$, and therefore, by Theorem 3.5 .

$$
\boldsymbol{A} \in \mathrm{TN}_{2,(n-1)^{2} \cdot 1}(\mathbb{K}) \subset \mathrm{TN}_{2,(\min (n, m)-1)^{2}}(\mathbb{K}) \subset \mathrm{TN}(\mathbb{K}) .
$$

Suppose now that $n=m, \boldsymbol{B} \in \mathrm{TP}_{2,(n-1)^{2}}(\mathbb{R})$ and that $\operatorname{per}(A) \neq \mathbb{0}$. We saw in Proposition 3.9 that $A$ is tropical diagonally dominant, in particular $A_{i i} \neq-\infty$, for all $i \in[n]$. After subtracting $A_{i i}$ to the $i$ th row of $A$, and dividing the $i$ th row of $\boldsymbol{B}$ by $\boldsymbol{B}_{i i}$, we may assume that $A_{i i} \equiv 0$ and $\boldsymbol{B}_{i i} \equiv 1$, so that $\boldsymbol{A}_{i i} \equiv 1$. Since $\boldsymbol{B} \in \mathrm{TP}_{2,(n-1)^{2}}$, we have $\boldsymbol{B} \in \mathrm{TP}_{2, C}$ for some $C<(n-1)^{2}$. We can write, as in Theorem 3.7 .

$$
\boldsymbol{A}=\boldsymbol{I}+\boldsymbol{B}^{\prime}
$$

where $\boldsymbol{I}$ is the identity matrix, and $\boldsymbol{B}^{\prime}$ has zero diagonal entries. From $\boldsymbol{B} \in \mathrm{TP}_{2, C}$, we deduce, using (3.8), that $\rho_{\max }\left(\boldsymbol{B}^{\prime}\right) \leqslant 1 / C^{1 / 2}$. Therefore, $\rho_{\max }(\boldsymbol{A}) \leqslant 1 / C^{1 / 2}<1 /(n-1)$. Then, it follows from Theorem 3.7 that $\operatorname{det} \boldsymbol{A}>0$. In particular, $\boldsymbol{A} \in \mathrm{GL}_{n}$.

Finally, if $A \in \mathrm{TN}^{\text {trop }}(\mathbb{R})$ and $\boldsymbol{B} \in \mathrm{TP}_{2,(n-1)^{2}}$, then, using Theorem 3.5] again, we deduce that $\boldsymbol{A} \in$ $\mathrm{TP}_{2,(n-1)^{2}}(\mathbb{K}) \subset \mathrm{TP}(\mathbb{K})$.

Remark 5.6. Let $\boldsymbol{A}$ and $A$ be as in Theorem [5.4 For all subsets $I \subset[n], J \subset[m]$ with the same cardinality, we denote by $\boldsymbol{A}_{I, J}$ the $I \times J$ submatrix of $\boldsymbol{A}$, and use a similar notation for the matrix $A$. Then, we note that if $A \in \mathrm{TN}^{\text {trop }}(\mathbb{R})$, and if $\boldsymbol{B} \in \mathrm{TN}_{2,(n-1)^{2}}(\mathbb{K})$ is choosen so that val $\boldsymbol{B}_{i j}$ is identically 0 , then,

$$
\text { val } \operatorname{det} \boldsymbol{A}_{I, J}=\operatorname{per} A_{I, J}
$$

Remark 5.7. Property (5.4) may not hold if we choose a lift different from the one of Theorem 5.4 For example

$$
A:=\left(\begin{array}{ll}
0 & 0 \\
0 & 0
\end{array}\right)=\operatorname{val} \boldsymbol{A}, \quad \text { where } \quad \boldsymbol{A}:=\left(\begin{array}{cc}
1 & 1 \\
1-t^{-1} & 1+t^{-1}
\end{array}\right)
$$


but the tropical permanent per $A=0$ differs from val $\operatorname{det} \boldsymbol{A}=2 t^{-1}$.

We get the following corollary of Theorem 5.4

Theorem 5.8. We have $\mathrm{TN}^{\text {trop }}=\operatorname{val}(\mathrm{TN})$.

Proof. We showed in Theorem 5.2 that $\operatorname{val}(\mathrm{TN}) \subset \mathrm{TN}^{\text {trop }}$. Conversely, suppose that $A \in \mathrm{TN}^{\text {trop }}$. Let us $C:=(n-1)^{2}$, and take any matrix $B \in \mathrm{TN}_{2, C}(\mathbb{R}) \cap \mathbb{R}_{>0}^{n \times m}$ (we already observed that such matrices exists for all $C$, for instance, $B$ may be a Vandermonde matrix). Then, it follows from Theorem 5.4 that $\boldsymbol{A}:=B * t^{A} \in \mathrm{TN}(\mathbb{K})$, and $\operatorname{val}(\boldsymbol{A})=A$.

The following is an immediate consequence of Theorem 5.8

Corollary 5.9. We have $\operatorname{TN}^{\text {trop }}(\mathbb{R})=\operatorname{val}\left(\operatorname{TN}\left(\mathbb{K}^{*}\right)\right)$.

Remark 5.10. Together with Corollary 4.8, Theorem 5.8 provides a polyhedral characterization of $\operatorname{val}(\mathrm{TN})$.

Remark 5.11. In [FJ11, Theorem 1.6.4] it is shown that every double echelon pattern is the Boolean pattern of some TN matrix. The above result can be recovered as a corollary of Theorem 5.4 Indeed, if $P$ is a Boolean matrix, we can define the tropical matrix $A$ such that $A_{i j}=0$ if $P_{i j}=1$, and $A_{i j}=-\infty$ if $P_{i j}=0$. If $P$ has a double echelon pattern, then $A$ belongs to $\mathrm{TN}_{2}^{\text {trop }}=\mathrm{TN}^{\text {trop }}$. Then, choosing any $\boldsymbol{B} \in \mathrm{TP}_{2,(n-1)^{2}}(\mathbb{R})$, we get that the matrix $\boldsymbol{A}$ such that $\boldsymbol{A}_{i j}=t^{A_{i j}} \boldsymbol{B}_{i j}$ belongs to TN $(\mathbb{K})$. By substituting $t$ by a suitably large real number, we end up with a matrix which is in $\operatorname{TN}(\mathbb{R})$ and which has the same pattern as the matrix $P$.

Conversely, Proposition 4.3 implies that if $\boldsymbol{A}$ is a TN matrix without zero row or column, then, the pattern of $\boldsymbol{A}$ is double echelon, recovering [FJ11, Coro. 1.6.5].

Remark 5.12. The relation between the set of double echelon matrices and the set of tropical totally nonnegative matrices can be understood in terms of nonarchimedean amoebas of a semialgebraic set, defined in $\$ 2$, Consider the semi-algebraic set $\mathcal{S} \subset \mathbb{R}^{n \times m}$ consisting of totally nonnegative matrices with no zero row or column, over a field $\mathscr{K}$. Let us first take $\mathscr{K}=\mathbb{R}$, equipped with the trivial valuation, val, which sends any non-zero element to 0 , and 0 to $-\infty$. Then, the results of [FJ11] which we just recalled mean that $\operatorname{val}(\mathcal{S})$ is the set of matrices with a double echelon pattern. If we take $\mathscr{K}=\mathbb{K}$, the field of Puiseux series, with the standard nonarchimedean valuation val, and if we consider $\mathcal{S}:=\operatorname{TP}(\mathbb{K})$, Theorem 3.4 characterizes the amoeba of val $(\mathcal{S})$. In this way, classical results made in combinatorial matrix theory, concerning Boolean patterns, appear to be related with tropical results: they all concern nonarchimedean amoebas, albeit with different valuations.

Corollary 5.13. $\operatorname{val}(\mathrm{TP})=\operatorname{val}\left(\mathrm{TN}\left(\mathbb{K}^{*}\right)\right)$.

Proof. Trivially, $\operatorname{val}(\mathrm{TP}) \subset \operatorname{val}\left(\mathrm{TN}\left(\mathbb{K}^{*}\right)\right)$. By Theorem 5.8 , we have $\operatorname{val}\left(\mathrm{TN}\left(\mathbb{K}^{*}\right)\right) \subset \mathrm{TN}^{\text {trop }}(\mathbb{R})$, and by the last statement in Theorem [5.4, for every matrix $A \in \mathrm{TN}^{\text {trop }}(\mathbb{R})$, we can find $\boldsymbol{A} \in \mathrm{TP}$ such that $\operatorname{val} \boldsymbol{A}=A$, showing that $\mathrm{TN}^{\text {trop }}(\mathbb{R}) \subset \operatorname{val}(\mathrm{TP})$.

We conclude from Theorem 5.2 and Theorem 5.4 that the set $\left\{A \in \mathrm{TN}^{\operatorname{trop}}: \operatorname{per}(A) \neq \mathbb{0}\right\}$ coincides with the set of matrices generated by tropical Jacobi matrices.

The following theorem summarizes our results.

Theorem C. The different classes of matrices considered so far satisfy the relations shown in Tables 1 and 国.

Proof. This follows from Theorem 3.4, Proposition 3.9, Theorem 4.6, Theorem 5.2, Theorem 5.4, Theorem 5.8 and Corollary 5.13 .

Remark 5.14. The results of Theorem C imply that

$$
\operatorname{val}\left(\mathrm{GL} \cap \mathrm{TN}\left(\mathbb{K}^{*}\right)\right)=\operatorname{val}(\mathrm{GL} \cap \mathrm{TN}) \cap \mathbb{R}^{n \times n}=\operatorname{val}(\mathrm{TP})
$$

Indeed, we have TP $\subset \mathrm{GL} \cap \mathrm{TN}\left(\mathbb{K}^{*}\right)$, and so, $\operatorname{val}(\mathrm{TP}) \subset \operatorname{val}\left(\mathrm{GL} \cap \mathrm{TN}\left(\mathbb{K}^{*}\right)\right)$. The inclusion $\operatorname{val}(\mathrm{GL} \cap$ $\left.\operatorname{TN}\left(\mathbb{K}^{*}\right)\right) \subset \operatorname{val}(\mathrm{GL} \cap \mathrm{TN}) \cap \mathbb{R}^{n \times n}$ is trivial. Now, if $M \in \operatorname{val}(\mathrm{GL} \cap \mathrm{TN}) \cap \mathbb{R}^{n \times n}$, we have $M=\operatorname{valM}$ with $\mathbf{M} \in \mathrm{TN}\left(\mathbb{K}^{*}\right)=\operatorname{val}(\mathrm{TP})$, showing that $\operatorname{val}(\mathrm{GL} \cap \mathrm{TN}) \cap \mathbb{R}^{n \times n} \subset \operatorname{val}(\mathrm{TP})$. 


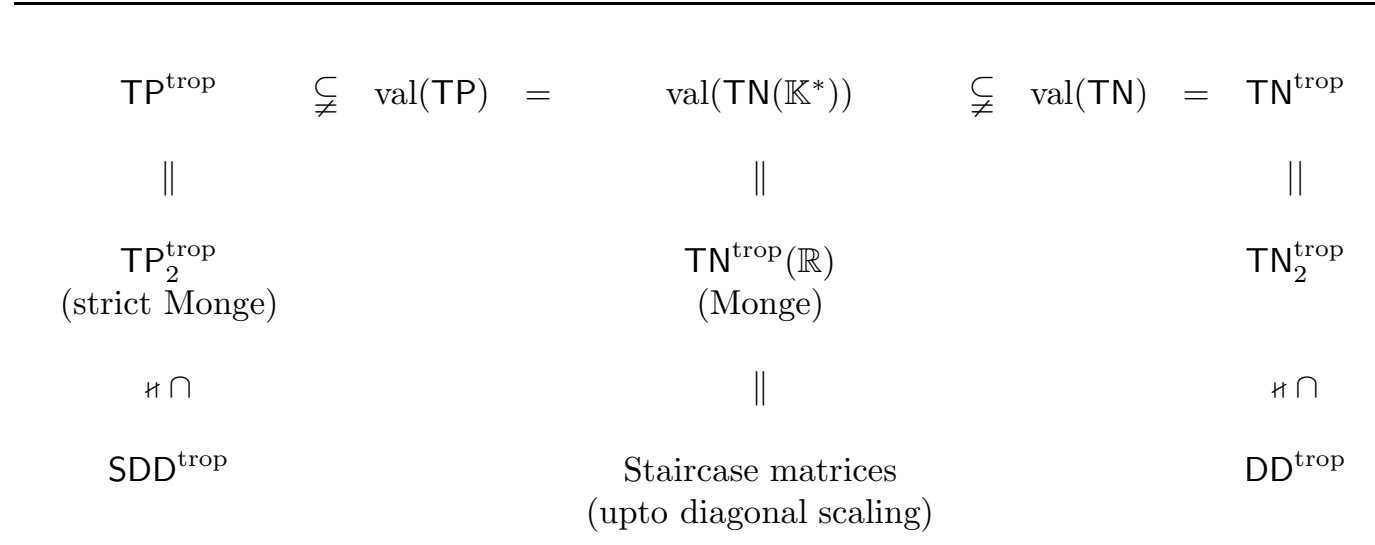

TABLE 1. Images by the valuation of nonarchimedean totally nonnegative matrices compared with tropical totally nonnegative matrices

$$
\begin{aligned}
& \operatorname{val}(\mathrm{TP}) \varsubsetneqq \operatorname{val}(\mathrm{GL} \cap \mathrm{TN}) \quad=\langle\text { tropical Jacobi matrices }\rangle \varsubsetneqq \operatorname{val}(\mathrm{TN}) \\
& \| \\
& \left\{A \in \mathrm{TN}^{\text {trop }}: \operatorname{per}(A) \neq \mathbb{O}\right\}
\end{aligned}
$$

TABLE 2. The special case of square matrices

Remark 5.15. Any subset $S$ of $\mathbb{R}_{\max }^{n \times n}$ can be decomposed in strata, consisting of those matrices in the set $S$ that share the same pattern (i.e., whose finite entries are in prescribed positions). It follows from Theorem 5.2 and (5.5) that

$$
\langle\text { tropical Jacobi matrices }\rangle \cap \mathbb{R}^{n \times n}=\text { Monge matrices }
$$

providing a polyhedral characterization of the main stratum of the semigroup 〈tropical Jacobi matrices〉, consisting of matrices with finite entries. This semigroup has other strata which it may be interesting to characterize.

Remark 5.16. Recall that an (elementary) Gaussian matrix differs from the identity matrix by at most one entry, that is non-zero. Analogously, an (elementary) tropical Gaussian matrix differs from the tropical identity matrix by at most one entry, that is finite. In particular, every (tropical) Jacobi matrix is a (tropical) Gaussian matrix. We characterized in Theorem C the multiplicative semigroup generated by tropical Jacobi matrices. It is an interesting open question to characterize the semigroup 〈Tropical Gaussian matrices〉. The case of of $3 \times 3$ matrices is solved in [Niv14, Lemma 4.5], stating that a matrix $A \in \mathbb{R}_{\max }^{3 \times 3}$ is not factorizable as a product of an invertible matrix and of Gaussian matrices if and only if there exists a fixed point-free permutation $\sigma \in S_{3}$ satisfying

$$
A_{i, \sigma(i)}<A_{i, k} A_{k, \sigma(i)}, k \neq i, \sigma(i), \quad \forall i .
$$

\section{Characteristic polynomial and eigenvalues of tropical totally nonnegative MATRICES}

Gantmacher and Krein established the following property: a totally nonnegative matrix has nonnegative eigenvalues, which are distinct and positive when the matrix is totally positive, see GK35. and also [FJ11, §5.2] for a more recent treatment. The field $\mathbb{K}$ is real closed and the theorem of Tarski, 
which we recalled in Section 2, shows that the first order theory of real closed fields is complete. It follows that the property found of Gantmacher and Krein remains valid for matrices with entries in $\mathbb{K}$.

We shall see that the tropical eigenvalues of a $\mathrm{TN}^{\text {trop }}$ matrix $A$ coincide with its diagonal entries, and that they coincide with the images by the valuation of the eigenvalues of an arbitrary TN lift of $A$. However, the tropical eigenvalues are not necessarily distinct when $A \in \mathrm{TP}^{\text {trop }}$.

We recall that the characteristic polynomial of $\boldsymbol{A} \in \mathbb{K}^{n \times n}$ is defined by

$$
\boldsymbol{f}_{\boldsymbol{A}}(\lambda)=\operatorname{det}(\lambda \boldsymbol{I}-\boldsymbol{A})=\lambda^{n}+\sum_{i=0}^{n-1}(-1)^{n-i} \boldsymbol{\alpha}_{n-i} \lambda^{i},
$$

where $\boldsymbol{I}$ is the identity matrix and $\boldsymbol{\alpha}_{k}$ is the sum of all the $k \times k$ principal minors of $\boldsymbol{A}$, with $\boldsymbol{\alpha}_{0}:=1$.

Similarly, the tropical characteristic polynomial of $A \in \mathbb{R}_{\max }^{n \times n}$ is defined as

$$
f_{A}(x)=\operatorname{per}(x \odot I \oplus A)=x^{\odot n} \oplus \bigoplus_{i=0}^{n-1} a_{n-i} \odot x^{\odot i},
$$

where $I$ is the tropical identity matrix, $a_{k}$ is the maximum between the weights of all $k \times k$ principal tropical minors of $A$, and $a_{0}:=\mathbb{1}$.

In But03] Butkovic discussed connections between max-algebraic problems and combinatorial optimization problems. He observed that computing the coefficient $a_{k}$ of the tropical characteristic polynomial is equivalent to a modification of the optimal assignment problem, the optimal principal submatrix problem, with an unsettled complexity in general. The next result shows that in the special case of $\mathrm{TN}^{\text {trop }}$ matrices, the $a_{k}$ are easy to compute.

Proposition 6.1. Suppose that $A \in \mathrm{TN}^{\text {trop }}$. Then, the coefficient $a_{k}$ of the tropical characteristic polynomial of $A$ coincides with the product of the $k$ largest diagonal entries of $A$.

Proof. Let us ordering the diagonal entries of $A$ from the smallest to greatest, we denote by $A_{i_{j}, i_{j}}$ the $j^{t h}$ greatest diagonal entry of $A$. That is, $A_{i_{1}, i_{1}} \geqslant A_{i_{2}, i_{2}} \geqslant \ldots \geqslant A_{i_{n}, i_{n}}$, where $\left\{i_{1}, \ldots, i_{n}\right\}=[n]$. From Proposition 3.9, $A \in \mathrm{DD}^{\text {trop }}$, and therefore, for every $I \subset[n]$ of cardinality $k$, we have per $A_{I I}=$ $\bigodot_{i \in I} A_{i i}$. Since $a_{k}$ is the maximum of all the terms of this form, we deduce that

$$
a_{k}=A_{i_{1} i_{1}} \odot \cdots \odot A_{i_{k} i_{k}} .
$$

The tropical roots of a tropical polynomial function are generally defined as the points of which the maximum of the monomials appearing in this polynomial is achieved twice at least. In particular, a finite tropical root is a nondifferentiability point of this function. We define tropical eigenvalue by specializing this definition to the characteristic polynomial.

Definition 6.2. We say that $\eta \in \mathbb{R}_{\max }$ is a tropical eigenvalue of $A$ if

$$
\exists i \neq j \text { s.t. } f_{A}(\eta)=a_{n-i} \odot \eta^{\odot i} \oplus a_{n-j} \odot \eta^{\odot j} .
$$

We say that $a_{k}$ is active if there exists an eigenvalue $\eta$ such that

$$
f_{A}(\eta)=a_{k} \odot \eta^{\odot n-k} .
$$

The multiplicity of a tropical eigenvalue $\eta \neq-\infty$ is the difference between the right derivative and left derivative of $f_{A}$ at this nondifferentiability point. If $-\infty$ is a tropical eigenvalue, its multiplicity is defined as the limit of of the derivative of $f_{A}$ at point $t$, as $t \rightarrow-\infty$.

Observe that counting multiplicities, an $n \times n$ tropical matrix $A$ has exactly $n$ eigenvalues.

Proposition 6.3. If $A \in \mathrm{TN}^{\text {trop }}$, then $a_{k}$ is active for all $k \in\{0\} \cup[n]$, and the tropical eigenvalues of $A$ (counting multiplicities) are precisely its diagonal entries.

Proof. It follows from (6.3) that

$$
f_{A}(x)=x^{\odot n} \oplus A_{i_{1} i_{1}} x^{\odot n-1} \oplus \cdots \oplus A_{i_{1} i_{1}} \odot \cdots \odot A_{i_{n} i_{n}} .
$$

Since $A_{i_{1}, i_{1}} \geqslant \ldots \geqslant A_{i_{n} i_{n}}$, the above polynomial function can be rewritten as

$$
f_{A}(x)=\left(x \oplus A_{i_{1} i_{1}}\right) \odot \cdots \odot\left(x \oplus A_{i_{n} i_{n}}\right) .
$$


Therefore, the $k$ th tropical eigenvalue of $A$ is given by $\eta_{k}=A_{i_{k} i_{k}}$. We also deduce that

$$
f_{A}\left(A_{i_{k}, i_{k}}\right)=a_{k} \odot A_{i_{k}, i_{k}}^{\odot n-k}
$$

showing that $a_{k}$ is active.

Proposition 6.5 below is illustrated by the following example.

Example 6.4. The characteristic polynomial $\boldsymbol{f}_{\boldsymbol{A}}(\lambda)=\left(\lambda-\left(t^{2}+t\right)\right)\left(\lambda-\left(t^{2}-t\right)\right)$ of

$$
\boldsymbol{A}=\left(\begin{array}{cc}
t^{2} & t \\
t & t^{2}
\end{array}\right) \in \mathrm{TP}
$$

has positive distinct roots. However, considering its valuation

$$
\operatorname{val}(\boldsymbol{A})=A=\left(\begin{array}{ll}
2 & 1 \\
1 & 2
\end{array}\right) \in \mathrm{TP}^{\text {trop }}
$$

we get the tropical characteristic polynomial $f_{A}(x)=x^{\odot 2} \oplus 2 \odot x \oplus 4$, with a tropical eigenvalue 2 of multiplicity 2. Nevertheless, the tropical coefficients and tropical eigenvalues are the corresponding images by the valuation of the coefficients and eigenvalues of $\boldsymbol{A}$. This may fail for $\boldsymbol{A} \in$ TP with $\operatorname{val}(\boldsymbol{A}) \notin \mathrm{TP}^{\text {trop }}$, as seen by the following example.

The valuation of the coefficients of the characteristic polynomial $\lambda^{2}-(t+2) \lambda+1$ of

$$
\boldsymbol{A}=\left(\begin{array}{cc}
t+1 & t \\
1 & 1
\end{array}\right) \in \mathrm{TP}
$$

do not coincide with the coefficients of the tropical characteristic polynomial $x^{\odot 2} \oplus 1 \odot x \oplus 1$ of

$$
\operatorname{val}(\boldsymbol{A})=\left(\begin{array}{cc}
1 & 1 \\
0 & 0
\end{array}\right) \notin \mathrm{TP}^{\text {trop }}
$$

Denote the characteristic polynomial of $A \in\left(\operatorname{TP}^{\operatorname{trop}}\right)^{n \times n}$ as in (6.2), with the tropical eigenvalues $\eta_{1} \geqslant \ldots \geqslant \eta_{n}$. By Theorem 5.3. we know that $A \in \mathrm{TP}^{\text {trop }}$ if and only if every lift $\boldsymbol{A}$ of $A$ is in TP. We denote the characteristic polynomial of $\boldsymbol{A}$ as in (6.1), and we denote by $\boldsymbol{\rho}_{1}>\cdots>\boldsymbol{\rho}_{n}$ the eigenvalues of $\boldsymbol{A}$.

The next result characterizes the valuation of the coefficients of the characteristic polynomial and of the eigenvalues of a totally positive matrix $\boldsymbol{A}$ with entries in $\mathbb{K}$.

Proposition 6.5. If $\boldsymbol{A} \in \mathrm{TP}$ is such that $A=\operatorname{val}(\boldsymbol{A}) \in \mathrm{TP}^{\text {trop }}$, we have

$$
\begin{array}{ll}
\operatorname{val}\left(\boldsymbol{\alpha}_{k}\right)=a_{k} & , \quad \forall k \in\{0\} \cup[n] . \\
\operatorname{val}\left(\boldsymbol{\rho}_{i}\right)=\eta_{i} & , \quad \forall i \in[n] .
\end{array}
$$

Proof. Recall that applying the valuation to strict inequalities over $\mathbb{K}_{\geqslant 0}$ yields weak inequalities.

We prove (6.4). In every submatrix $M$ of the lift $\boldsymbol{A}$ of $A$, the valuation of product of diagonal entries of $M$ is the only one of maximal weight in $\operatorname{val}(\operatorname{det}(M))$. Since $\boldsymbol{\alpha}_{k}$ is the sum of $k \times k$ principal minors of $\boldsymbol{A}$, we get that $\operatorname{val}\left(\boldsymbol{\alpha}_{0}\right)=a_{0}$ and for every $k \in[n]$

$$
\begin{aligned}
\operatorname{val}\left(\boldsymbol{\alpha}_{k}\right) & =\max _{\substack{I \subset[n]: \\
|I|=k}} \operatorname{val}\left(\sum_{\sigma \in S_{I}} \operatorname{sgn}(\sigma) \prod_{i \in I} \boldsymbol{A}_{i, \sigma(i)}\right)=\max _{\substack{I \subset[n]: \\
|I|=k}}\left\{\operatorname{val}\left(\prod_{i \in I} \boldsymbol{A}_{i, i}\right)\right\} \\
& =\max _{\substack{I \subset[n]: \\
|I|=k}}\left\{\sum_{i \in I} \operatorname{val}\left(\boldsymbol{A}_{i, i}\right)\right\}=\bigoplus_{\substack{I \subset[n]: \\
|I|=k}} \bigodot_{i \in I} A_{i, i}=\bigoplus_{\substack{I \subset[n]: \\
|I|=k}} \bigoplus_{\sigma \in S_{I}} \bigodot_{i \in I} A_{i, \sigma(i)}=a_{k} .
\end{aligned}
$$

We now prove (6.5). By Puiseux Theorem, we know that the image by the valuation of the roots of the polynomial $\boldsymbol{f}_{\boldsymbol{A}}(\lambda)$ are precisely the slopes of the associated Newton polytope, defined as the upper boundary of the concave hull of the points $\left(i, \operatorname{val}\left(\boldsymbol{\alpha}_{n-i}\right)\right), i=0, \ldots, n$. By Legendre-Fenchel duality, these are precisely the tropical roots of

$$
x \mapsto \max _{i \in[n]}\left\{i x+\operatorname{val}\left(\boldsymbol{\alpha}_{i}\right)\right\},
$$


counted with multiplicities. In general $\operatorname{val}\left(\boldsymbol{\alpha}_{i}\right) \leqslant a_{i}$, but equality holds when $A \in \mathrm{TP}^{\text {trop }}$, as seen in the first item. Therefore, the tropical eigenvalues of $A$ coincides with the tropical roots of the polynomial function (6.6).

Remark 6.6. In general, it is known that the sequence of valuations of the eigenvalues of a matrix $\boldsymbol{A}$ with entries in $\mathbb{K}$ is weakly majorized by the sequence of tropical eigenvalues of the matrix obtained by applying the valuation to every entry of $\boldsymbol{A}$, see Theorem 4.4 of ABG16. The previous result shows that equality holds when $\boldsymbol{A} \in \mathrm{TP}$.

Remark 6.7. We saw that the eigenvalues of a tropical totally nonnegative matrix have a simple characterization. We may also consider eigenvectors, i.e., solutions $u$ of $A u=\lambda u$ for some scalar $\lambda$. Such eigenvectors can be determined, by exploiting the general combinatorial characterization of tropical eigenvectors, in terms of shortest paths matrix (see for instance [But10, § 4.2]). It may be interesting to investigate whether eigenvectors show more structure in the tropical totally positive case.

\section{Other ASPECTS OF tropical total POSitivity}

7.1. Tropical totally positive matrices and the tropical totally positive Grassmannian. We next relate the set of totally positive matrices with the totally positive tropical Grassmannian considered by Postnikov, Speyer and Williams [Pos06, SW05].

To any $n \times m$ matrix $\boldsymbol{A}$ with entries in a field, we associate a vector of Plücker coordinates, whose entries are the maximal minors. We denote by $\Delta(\boldsymbol{A})$ the vector of Plücker coordinates of this matrix, assuming without loss of generality that $n \leqslant m$. So, $\Delta(\boldsymbol{A})$ is a vector of size $C_{m}^{n}$ whose entries are indexed by the subsets $I \subset[m]$ such that $|I|=n$, ordered lexicographically. We denote the $I$-coordinate of $\Delta(\boldsymbol{A})$ by $\Delta_{I}(\boldsymbol{A}):=\operatorname{det} \boldsymbol{A}_{I}$, where $\boldsymbol{A}_{I}$ is the maximal submatrix of $\boldsymbol{A}$ of column set $I$. If $A$ is a $n \times m$ tropical matrix, the tropical Plücker coordinates are defined analogously. We still denote them by $\Delta(A)$. In particular, we have $\Delta_{I}(A)=\operatorname{per}\left(A_{I}\right)$, where now per denotes the tropical permanent of the maximal submatrix $A_{I}$.

The Grassmannian $\mathrm{Gr}_{k, n}$ on $\mathbb{K}$ is the space of $k$-dimensional subspaces of $\mathbb{K}^{n}$. An element of $\mathrm{Gr}_{k, n}$ can be represented by an $k \times n$ matrix of full-rank, modulo left multiplication by $\mathrm{GL}_{k}$. The map $\boldsymbol{A} \mapsto$ $\Delta(\boldsymbol{A})$ yields an embedding $\operatorname{Gr}_{k, n} \hookrightarrow \mathbb{P}^{\left(\begin{array}{l}n \\ k\end{array}\right)-1}(\mathbb{K})$. The totally positive Grassmannian $\mathrm{Gr}_{k, n}^{+} \subset \mathrm{Gr}_{k, n}$, studied in Pos06, SW05, is the subset of $k$-dimensional subspaces that can be represented by matrices $\boldsymbol{A} \in \mathbb{K}^{k \times n}$ with $\Delta_{I}(\boldsymbol{A})>0$ for all subsets $I$ of $[n]$ with $k$ elements. The totally nonnegative Grassmannian $\mathrm{Gr}_{k, n}^{\geqslant 0} \subset \mathrm{Gr}_{k, n}$ is defined in a similar way, requiring this time that $\Delta_{I}(\boldsymbol{A}) \geqslant 0$ for all subsets $I$ of $[n]$ with $k$ elements.

There is a known correspondence between the matrices of $\boldsymbol{B} \in \mathrm{TP}^{k \times(n-k)}$ and the elements of the totally positive Grassmannian $\mathrm{Gr}_{k, n}^{+}$. To see this, we first associate with $\boldsymbol{B}$ the matrix

$$
\imath(\boldsymbol{B}):=\left(\begin{array}{ccccccc}
1 & \ldots & 0 & 0 & (-1)^{k-1} b_{k, 1} & \ldots & (-1)^{k-1} b_{k, n-k} \\
\vdots & \ddots & \vdots & \vdots & \vdots & \ddots & \vdots \\
0 & \ldots & 1 & 0 & -b_{2,1} & \ldots & -b_{2, n-k} \\
0 & \ldots & 0 & 1 & b_{1,1} & \ldots & b_{1, n-k}
\end{array}\right) .
$$

In other words, $\imath(\boldsymbol{B})=(\mathcal{I} \mid \tilde{\boldsymbol{B}}) \in \mathbb{K}^{k \times n}$, where $\mathcal{I}$ is the $k \times k$ identity matrix, and the matrix $\tilde{\boldsymbol{B}}$ is defined by $\tilde{\boldsymbol{B}}_{t}=(-1)^{k-t} \boldsymbol{B}_{k-t+1}$, for all $t \in[k]$.

One can check that

$$
\operatorname{det}\left(\boldsymbol{B}_{I, J}\right)=\Delta_{([k] \backslash \tilde{I}) \cup \tilde{J}}(\imath(\boldsymbol{B})),
$$

where $\boldsymbol{B}_{I, J}$ denotes the $I \times I$ submatrix of $\boldsymbol{B}, \tilde{I}=\{k-t+1: t \in I\}$, and $\tilde{J}=\{t+k: t \in J\}$. We consider the following map

$$
\phi: \boldsymbol{B} \mapsto \Delta(\imath(\boldsymbol{B})) .
$$

Postnikov made the following observation in Pos06, up to a trivial modification (the entries of the matrix $b$ are listed from bottom to top in (7.1)).

Proposition 7.1 (See Prop. 3.10 of [Pos06]). The map $\phi$ sends bijectively $\operatorname{TP}^{k \times(n-k)}$ to the totally positive Grassmannian $\mathrm{Gr}_{k, n}^{+}$. 
We next compare the set of tropical totally positive matrices with the tropical totally positive Grassmannian $\operatorname{Trop}^{+}(\mathrm{Gr}):=\operatorname{val}\left(\mathrm{Gr}_{n, k}^{+}\right)$, studied in [SW05. Here, $\operatorname{Trop}^{+}(\mathrm{Gr})$ is thought of as the subset of the tropical projective space $\mathbb{P}^{C_{n}^{k}-1}\left(\mathbb{R}_{\max }\right)$, obtained as the image by the valuation of $\mathrm{Gr}_{n, k}^{+}$, the latter being identified to its image by the Plücker embedding.

We now associate to a matrix $B \in \mathbb{R}^{k \times(n-k)}$ the matrix

$$
\imath(B):=\left(\begin{array}{ccccccc}
0 & \ldots & -\infty & -\infty & b_{k, 1} & \ldots & b_{k, n-k} \\
\vdots & \ddots & \vdots & \vdots & \vdots & \ddots & \vdots \\
-\infty & \ldots & 0 & -\infty & b_{2,1} & \ldots & b_{2, n-k} \\
-\infty & \ldots & -\infty & 0 & b_{1,1} & \ldots & b_{1, n-k}
\end{array}\right)
$$

and define the map $\phi: \mathbb{R}^{k \times(n-k)} \rightarrow \mathbb{R}^{C_{n}^{k}}$, such that $\phi(B)$ is the vector with entries

$$
\phi_{I}(B)=\operatorname{per}(\imath(B))_{I}
$$

for any subset $I$ of $[n]$ with $k$ elements. The next result shows that in the tropical setting, the map $\phi$ is still injective. We shall see in Example 7.4 that it is no longer surjective.

Proposition 7.2. The map $\phi$ sends linearly $\left(\mathrm{TN}^{\mathrm{trop}}(\mathbb{R})\right)^{k \times(n-k)}$ to a closed polyhedral subset of $\operatorname{Trop}^{+}\left(\mathrm{Gr}_{n, k}\right)$.

Proof. The construction of Theorem 5.4 implies that any matrix $B \in \mathrm{TN}^{k \times(n-k)}(\mathbb{R})$ has a lift $\boldsymbol{B} \in \mathrm{TP}$, meaning that $B=\operatorname{val}(\boldsymbol{B})$. Moreover, by (5.4), this lift can be chosen such that

$$
\text { per } B_{I, J}=\text { val } \operatorname{det} \boldsymbol{B}_{I, J}
$$

for all subsets $I \subset[k], J \subset[n-k]$ that have the same cardinality. Then, by Proposition $7.1, \phi(\boldsymbol{B}) \in$ $\mathrm{Gr}_{k, n}^{+}$. Note also that

$$
\operatorname{per}\left(B_{I, J}\right)=\Delta_{([k] \backslash \tilde{I}) \cup \tilde{J}}(\imath(B)) .
$$

We deduce from (7.4) and (7.5) that $\phi(B)=\operatorname{val} \phi(\imath(\boldsymbol{B})) \in \operatorname{Trop}^{+} \operatorname{Gr}_{k, n}$. Moreover, if $B, B^{\prime}$ are distinct elements of $\mathrm{TN}^{k \times(n-k)}(\mathbb{R})$, then, it follows from (7.5) that $\phi(B)$ and $\phi\left(B^{\prime}\right)$ have at least one distinct coordinate. Observe that $\phi(B)$ and $\phi\left(B^{\prime}\right)$ have also one identical coordinate, namely, the one corresponding to the choice $I=J=\varnothing$ in (7.5). It follows that the vectors $\phi(B)$ and $\phi\left(B^{\prime}\right)$ are non proportional in the tropical sense, and so, they define distinct elements of $\operatorname{Trop}^{+}\left(\operatorname{Gr}_{k, n}\right)$. We already observed that for a square matrix $C$ in $\operatorname{TN}(\mathbb{R})$, the maximum in evaluating per $C$ is always achieved on the diagonal. It follows that the map $\phi$ restricted to $\mathrm{TN}(\mathbb{R})$ is linear (it is only piecewise linear in $\mathbb{R}^{k \times(n-k)}$ ). Hence, this map sends the set $\mathrm{TN}(\mathbb{R})$, which is polyhedral, to a closed polyhedron contained in $\operatorname{Trop}^{+}\left(\mathrm{Gr}_{k, n}\right)$.

Example 7.3. Consider the following instance of the construction (7.3):

$$
B=\left(\begin{array}{cc}
0 & -2 \\
0 & -1 \\
0 & 0
\end{array}\right) \in \mathrm{TP}^{\text {trop }}, \quad A:=\imath(B)=\left(\begin{array}{ccccc}
0 & -\infty & -\infty & 0 & 0 \\
-\infty & 0 & -\infty & 0 & -1 \\
-\infty & -\infty & 0 & 0 & -2
\end{array}\right),
$$

The image of $B$ by the map $\phi$ is the point of the tropical projective space with coordinates

$$
\Delta(A)=(0: 0:-2: 0:-1:-1: 0: 0: 0: 0)
$$

Proposition 7.2 shows that this point belongs to $\operatorname{Trop}^{+} \mathrm{Gr}_{3,5}$. This can be checked by elementary means as follows. Since $B \in \mathrm{TP}^{\text {trop }}$, we do not need to consider the special lift of Theorem 5.4, any lift of $B$, and in particular the trivial lift $\boldsymbol{B}=\left(t^{B_{i j}}\right)$, belongs to $\operatorname{TP}(\mathbb{K})$. Then, the matrix $\boldsymbol{B}$ is sent to the following matrix by the construction (7.1)

$$
\imath(\boldsymbol{B})=\left(\begin{array}{ccccc}
1 & 0 & 0 & 1 & 1 \\
0 & 1 & 0 & -1 & -t^{-1} \\
0 & 0 & 1 & 1 & t^{-2}
\end{array}\right) .
$$

One can check that

$$
\Delta(\imath(\boldsymbol{B}))=\left(1: 1: t^{-2}: 1: t^{-1}: t^{-1}-t^{-2}: 1: 1: 1-t^{-2}: 1-t^{-1}\right) \in \mathrm{Gr}_{3,5}^{+}
$$


and so, $\operatorname{val} \Delta(\imath(\boldsymbol{B}))=\Delta(A) \in \operatorname{Trop}^{+} \mathrm{Gr}_{3,5}$.

Example 7.4. We next give an elementary example showing that $\phi\left(\left(\mathrm{TN}^{\mathrm{trop}}\right)^{2 \times 2}\right)$ is a strict subset of Trop ${ }^{+} \mathrm{Gr}_{2,4}$. Consider the matrix

$$
D=\left(\begin{array}{cccc}
0 & -1 & -2 & -3 \\
0 & 0 & 0 & 0
\end{array}\right)
$$

and the trivial lift $\mathbf{D}:=\left(t^{D_{i j}}\right)$. It can be checked that

$$
\Delta(\mathbf{D})=\left(1-t^{-1}: 1-t^{-2}: 1-t^{-3}: t^{-1}-t^{-2}: t^{-1}-t^{-3}: t^{-2}-t^{-3}\right) \in \mathrm{Gr}_{2,4}^{+}
$$

and so, $\operatorname{val}(\Delta(\mathbf{D}))=\Delta(D)=(0: 0: 0:-1:-1:-2) \in \operatorname{Trop}^{+} \operatorname{Gr}_{2,4}$. However, this element of Trop ${ }^{+} \mathrm{Gr}_{2,4}$ does not belong to the image of $\mathrm{TN}^{\text {trop }}(\mathbb{R})$ by the map $\phi$. Indeed, assume by contradiction that $\Delta(D)=\phi(C)$ for some matrix $C$ of size $2 \times 2$. Using (7.5), we get that $0=\Delta_{\{1,3\}}(D)=C_{1,1}$, $0=\Delta_{\{1,4\}}(D)=C_{1,2},-1=\Delta_{\{2,3\}}(D)=C_{2,1},-1=\Delta_{\{2,4\}}(D)=C_{22}$, and so

$$
C=\left(\begin{array}{cc}
0 & 0 \\
-1 & -1
\end{array}\right) \text {. }
$$

However, using again (7.5), we see that $-2=\Delta_{\{3,4\}}(D)=$ per $C_{\{1,2\},\{1,2\}}=-1$, a contradiction.

Remark 7.5. The question of characterizing the image of the map $\phi$ has already been studied without considerations of total positivity. Fink and Rincón called "tropical Stiefel map" [FR15] the map $A \mapsto \Delta(A)$ sending a tropical matrix to the vector of its maximal tropical minors. The image $\Delta\left(\mathbb{R}^{k \times n}\right)$ is called the Stiefel image. Rincón and Fink observed that

$$
\phi\left(\mathbb{R}^{k \times(n-k)}\right) \subset \Delta\left(\mathbb{R}^{k \times n}\right) \subset \operatorname{val}\left(\mathrm{Gr}_{n, k}(\mathbb{K})\right) .
$$

They showed that the second inclusion is strict for $(k, n)=(2,6)$. This also follows from a result of Herrmann, Joswig and Speyer, see Corollary 14 and Example 10 in HJS14. Corollary 3.8 of [FR15] also allows one to represent $\Delta\left(\mathbb{R}^{k \times n}\right)$ as the union of the images of a family of maps, including the map $\phi$ as a special case. Hence, this result implies that the first inclusion in (7.6) is also strict. We leave it for further investigation to look for refinements of these results in the case of the tropical totally positive Grassmannian.

Remark 7.6. A related issue is to investigate which part of the totally nonnegative tropical Grassmanian is given by the image $\phi\left(\mathrm{TN}^{\text {trop }}\right)$, i.e., by Plücker coordinates of the form $\phi(A)$ where now the matrix $A \in \mathrm{TN}^{\text {trop }}$ (a tropical totally nonnegative matrix with possibly $-\infty$ entries). It may be interesting in particular to investigate relations with Postnikov's positive Grassman cells. Recall that Postnikov introduced in [Pos06, Section 3] a decomposition of the totally nonnegative Grassmannian in terms of "positive Grassman cells". Such a cell is associated to a matroid $\mathcal{M}$; it consists of those elements of $\mathrm{Gr}_{k, n}^{+}$represented by a matrix $\boldsymbol{A}$, such that $\Delta_{I}(\boldsymbol{A})$ is nonzero if and only $I$ is a basis of the matroid $\mathcal{M}$. The special matroids corresponding to nonempty cells are called positroids.

Remark 7.7. Suppose $A \in\left(\mathrm{TN}^{\text {trop }}\right)^{n \times m}$ is in double echelon form, with $n \leqslant m$, and that it has signnonsingular tropical Plücker coordinates. Then, there exists $B \in\left(\mathrm{TP}^{\mathrm{trop}}\right)^{n \times m}$ s.t. $A$ and $B$ represent the same element in $\operatorname{Trop}^{+} \mathrm{Gr}_{n, m}$.

We construct the matrix $B$ as follows. First, we set $B_{i, j}=A_{i, j}$, for all $i \leqslant j \leqslant m-n+i$ with $i \in[n]$ (in other words, the diagonal entries of all the maximal submatrices of $A$ are unchanged). Since $A \in \mathrm{TN}^{\text {trop }}$, the identity permutation in every maximal minor of $A$ is of maximal weight. We deduce that every $2 \times 2$ solid submatrix $A_{\{i, i+1\},\{j, j+1\}}=B_{\{i, i+1\},\{j, j+1\}}$ such that $i+1 \leqslant j \leqslant m-n+i-1$, must be sign-nonsingular. Indeed, the maximal submatrix in $A$ containing a solid $2 \times 2$ sign singular matrix must be sign-singular (there must be at least two permutations of maximal weight in the latter matrix, namely the identity, and a transposition). Then, for every $i \in[n]$ we construct the $B_{i, j} \neq \mathbb{0}$ successively for $j=i-1, \ldots, 2,1$, by requiring that $B_{\{i-1, i\},\{j, j+1\}}$ satisfies the strict Monge property, and then successively for $j=m-n+i+1, \ldots, m-1, m$, by requiring that $B_{\{i, i+1\},\{j-1, j\}}$ satisfies the strict Monge property. 
7.2. Tropical totally nonnegative matrices and planar networks. The combinatorial properties of minors of the weight matrix associated with a planar network are well known [FZ00]. These were studied by Karlin and McGregor back in the 50's (see [KM59]). Some applications were given by Gessel and Viennot in GV85, GV89. In this context, totally nonnegative matrices arise as weight matrices of planar networks. We next show that, as an immediate consequence of the previous result, the same is true in the tropical setting. To this end, we first recall or state some basic definitions.

A graph is called planar if it can be drawn on a plane so that its edges have only endpointintersections. A planar network is a weighted directed planar graph, with no cycles. Throughout, we assume a network has $n$ sources and targets, numbered bottom to top, with edges directed left to right assigned with real weights.

Let $G$ be a planar network with weights in $\mathbb{K}$. The weight of a path between nodes $i, j \in[n]$ in $G$ (if exists) is the product of weights of its edges. The weight matrix of $G$ is an $n \times n$ matrix having the sum of weights of all paths from $i$ to $j$ for its $i, j$-entry, and 0 if such a path does not exist.

Suppose now that the edges of $G$ are weighted by elements of $\mathbb{R}_{\max }$. Then, the tropical weight matrix of $G$ has the paths of maximal weight from $i$ to $j$ for its $i, j$-entry, and $-\infty$ if such a path does not exist, where the weight of a path becomes the sum of weights of its edges.

Example 7.8. For all $\alpha \leqslant 6$, the planar network

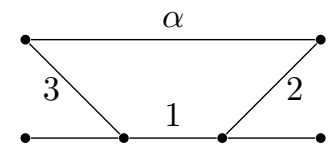

corresponds to the tropical weight matrix

$$
A=\left(\begin{array}{ll}
1 & 3 \\
4 & 6
\end{array}\right)
$$

Corollary 7.9. The tropical weight matrix of every planar network is tropical totally nonnegative, and every tropical totally nonnegative matrix is the tropical weight matrix of some planar network.

Proof. The weight matrix $\boldsymbol{A}$ of a planar network whose edges $e$ are weighted by nonnegative elements $\boldsymbol{w}_{e} \in \mathbb{K}$ is totally nonnegative (see e.g. [FZ00, Coro 2]). The valuation sends this weight matrix to the tropical weight matrix $A$ arising by weighting the edge $e$ with val $\boldsymbol{w}_{e}$. By Theorem [5.8 $A=\operatorname{val} \boldsymbol{A}$ is totally nonnegative.

Conversely, Theorem 5.2 shows that a finite tropical TN matrix $A$ can be factored as a product of tropical Jacobi matrices. A classical result allows one to identify a product of Jacobi matrices to the weight matrix of a planar network, see the discussion before Theorem 13 of [FZ00]. The same arguments works in the tropical setting, and we conclude that $A$ is the tropical weight matrix of a planar network.

\section{REFERENCES}

[ABG07] M. Akian, R. Bapat, and S. Gaubert. Max-plus algebras. In Leslie Hogben, editor, Handbook of linear algebra, Discrete Mathematics and its Applications (Boca Raton). CRC Press, Boca Raton, FL, second edition, 2007. Chapter 25.

[ABG16] M. Akian, R. Bapat, and S. Gaubert. Non-archimedean valuations of eigenvalues of matrix polynomials. Linear Algebra Appl., 498:592-627, 2016.

[ABGJ15] X. Allamigeon, P. Benchimol, S. Gaubert, and M. Joswig. Tropicalizing the simplex algorithm. SIAM J. Disc. Math., 29(2):751-795, 2015.

[AGG14] M. Akian, S. Gaubert, and A. Guterman. Tropical Cramer determinants revisited. In Tropical and idempotent mathematics and applications, volume 616 of Contemp. Math., pages 1-45. Amer. Math. Soc., Providence, RI, 2014. 
[AGS16a] X. Allamigeon, S. Gaubert, and M. Skomra. Solving generic nonarchimedean semidefinite programs using stochastic game algorithms. In Proceedings of the 41st International Symposium on Symbolic and Algebraic Computation (ISSAC). ACM, 2016.

[AGS16b] Xavier Allamigeon, Stéphane Gaubert, and Mateusz Skomra. Tropical spectrahedra, 2016. arXiv:1610.06746.

[AGS17] M. Akian, S. Gaubert, and M. Sharify. Log-majorization of the moduli of the eigenvalues of a matrix polynomial by tropical roots. Linear Algebra and Appl., 528:394-435, 2017.

[AKNR13] M. Avendano, R. Kogan, M. Nisse, and J. M. Rojas. Metric estimates and membership complexity for archimedean amoebae and tropical hypersurfaces, 2013.

[Ale13] D. Alessandrini. Logarithmic limit sets of real semi-algebraic sets. Adv. Geom, 13:155-190, 2013.

[And87] T. Ando. Totally positive matrices. Linear Algebra Appl., 90:165-219, 1987.

[BCOQ92] F. Baccelli, G. Cohen, G.J. Olsder, and J.P. Quadrat. Synchronization and linearity. Wiley Series in Probability and Mathematical Statistics: Probability and Mathematical Statistics. John Wiley \& Sons, Ltd., Chichester, 1992. An algebra for discrete event systems.

[BFZ96] A. Berenstein, S. Fomin, and A. Zelevinsky. Parametrizations of canonical bases and totally positive matrices. Adv. Math., 122(1):49-149, 1996.

[BKR96] R. E. Burkard, B. Klinz, and R. Rudolf. Perspectives of Monge properties in optimization. Discrete Appl. Math., 70(2):95-161, 1996

[BS95] R. A. Brualdi and B. L. Shader. Matrices of Sign-Solvable Linear Systems. Number 116 in Cambridge Tracts in Mathematics. Cambridge University Press, 1995.

[But03] P. Butkovič. Max-algebra: the linear algebra of combinatorics? Linear Algebra Appl., 367:313-335, 2003.

[But10] P. Butkovič. Max-linear systems: theory and algorithms. Springer Monographs in Mathematics. SpringerVerlag London, Ltd., London, 2010.

[Den86] J. Denef. p-adic semi-algebraic sets and cell decomposition. J. Reine Angew. Math., 369:154-166, 1986.

[DS04] M. Develin and B. Sturmfels. Tropical convexity. Doc. Math., 9:1-27, 2004.

[EKL06] M. Einsiedler, M. Kapranov, and D. Lind. Non-Archimedean amoebas and tropical varieties. J. Reine Angew. Math., 601:139-157, 2006.

[EP05] A. J. Engler and A. Prestel. Valued Fields. Springer Monogr. Math. Springer, Berlin, 2005.

[Fal99] S. M. Fallat. Totally nonnegative matrices. Phd dissertation, Department of Mathematics, College of William and Mary, Williamsburg, VA, 1999.

[Fie06] M. Fiedler. Subtotally positive and Monge matrices. Linear Algebra Appl., 413:177-188, 2006.

[FJ11] S. M. Fallat and C. R. Johnson. Totally Nonnegative Matrices. Princeton Series in Applied Mathematics. Princeton university press, 2011.

[FR15] A. Fink and F. Rincón. Stiefel tropical linear spaces. Journal of Combinatorial Theory, Series A, $135: 291$ $331,2015$.

[Fri86] S. Friedland. Limit eigenvalues of nonnegatives matrices. Linear Alg. and Appl., 74:173-178, 1986.

[FZ00] S. Fomin and A. Zelevinsky. Total positivity: Tests and parametrizations. The Mathematical Intelligencer, 22(1):23-33, 2000

[GB99] S. Gaubert and P. Butkovič. Sign-nonsingular matrices and matrices with unbalanced determinant in symmetrised semirings. Linear Algebra Appl., 301(1-3):195-201, 1999.

[GK35] F. R. Gantmacher and M. G. Krein. Sur les matrices oscillatoires. C. R. Acad. Sci. (Paris), 201:577-579, 1935.

[GKZ94] I. M. Gelfand, M. M. Kapranov, and A. V. Zelevinsky. Discriminants, Resultants, and Multidimensional Determinants. Mathematics: Theory \& Applications. Birkhäuser Boston, 1994.

[GM96] M. Gasca and C. A. Micchelli, editors. Total positivity and its applications, volume 359 of Mathematics and its Applications. Kluwer Academic Publishers Group, Dordrecht, 1996.

[GP95] M. Gasca and J. M. Pena. On the characterization of totally positive matrices. Advances in Computational Mathematics, 3(1):239-250, 1995.

[GV85] I. Gessel and G. X. Viennot. Binomial determinants, paths, and hooklength formulae. Adv. in Math., 58:300321, 1985.

[GV89] I. Gessel and G. X. Viennot. Determinants, paths, and plane partitions. preprint, 1989.

[HJS14] S. Herrmann, M. Joswig, and D. Speyer. Dressians, tropical Grassmannians, and their rays. Forum Mathematicum, 26(6):1853-1881, 2014.

[HR15] G. H. Hardy and M. Riesz. The general theory of Dirichlet's series. Cambridge University Press, Cambridge, 1915.

[IMS07] I. Itenberg, G. Mikhalkin, and E. Shustin. Tropical algebraic geometry, volume 35 of Oberwolfach Seminars. Birkhäuser Verlag, Basel, 2007.

[KM59] S. Karlin and G. McGregor. Coincidence probabilities. Pacific J. Math., 9:1141-I164, 1959.

[Lam14] T. Lam. Totally nonnegative grassmannian and grassmann polytopes. Int. Press, Somerville, MA, $2016,2014$.

[Loe55] C. Loewner. On totally positive matrices. Mathematische Zeitschrift, 63(1):338-340, 1955.

[Mar02] D. Marker. Model theory: an introduction. Springer, 2002.

[Mar10] T. Markwig. A field of generalised Puiseux series for tropical geometry. Rend. Semin. Mat. Univ. Politechnico di Torino, 68(1):79-92, 2010.

[MS15] D. Maclagan and B. Sturmfels. Introduction to tropical geometry. AMS Graduate Studies in Mathematics, $161,2015$. 
[Niv14] A. Niv. Factorization of tropical matrices. J. Algebra Appl., 13(1):1350066, 26, 2014.

[Pas89] J. Pas. Uniform $p$-adic cell decomposition and local zeta functions. J. Reine Angew. Math., 399:137-172, 1989.

[Plu90] M. Plus. Linear systems in (max, +)-algebra. In Proceedings of the 29th Conference on Decision and Control, Honolulu, Dec. 1990.

[Pos06] A. Postnikov. Total positivity, Grassmannians, and networks, 2006. arXiv:math/0609764

[Rib92] P. Ribenboim. Fields: algebraically closed and others. Manuscripta Math., 75:115-150, 1992.

[SS04] D. Speyer and B. Sturmfels. The tropical Grassmannian. Adv. Geom., 4:389- 411, 2004.

[SW05] D. Speyer and L. Williams. The tropical totally positive Grassmannian. J. Alg. Comb., 22(2):189-210, 2005.

[vdDS98] L. van den Dries and P. Speissegger. The real field with convergent generalized power series. Trans. Amer. Math. Soc., 350(11):4377-4421, 1998.

[Whi52] A. M. Whitney. A reduction theorem for totally positive matrices. J. d'Analyse Math., 2:88-92, 1952.

[Yu15] J. Yu. Tropicalizing the positive semidefinite cone. Proc. Amer. Math. Soc., 143:1891-1895, 2015.

Stéphane Gaubert, InRIA Saclay-Île-de-France and CMAP, École polytechnique, CNRS. Address: CMAP, École polytechnique, Route de Saclay, 91128 Palaiseau Cedex, France.

E-mail address: Stephane.Gaubert@inria.fr

Adi Niv, Mathematics Department, Science Faculty, Kibbutzim College. Address: Kibbutzim College, 149 Namir Rd., Tel-Aviv, IsRael.

E-mail address: adi.niv@smkb.ac.il 